\title{
TOPOLOGY OF LIE GROUPS AND CHARACTERISTIC CLASSES ${ }^{1}$
}

\begin{abstract}
ARMAND BOREL
1. Introduction. The notion of continuous group, later called Lie group, introduced by S. Lie in the nineteenth century, has classically a local character. Although global Lie groups were also sometimes considered, it is only after 1920 that this concept was clearly formulated. We recall that a Lie group in the large is first a manifold, i.e., a topological Hausdorff space admitting a covering by open sets, each of which is homeomorphic to euclidian $n$-space; second it is a group; third it is a topological group, i.e., the product $x \cdot y$ of $x$ and $y$ and the inverse $x^{-1}$ are continuous functions of their arguments; and finally it is required that there exist coordinates in a neighbourhood $V$ of the identity element $e$ such that if $x, y$, and $x \cdot y$ are in $V$, the coordinates of $x \cdot y$ are analytic functions in the coordinates of $x$ and $y$. Gleason, Montgomery, and Zippin recently proved that the last condition follows from the others, thus solving Hilbert's fifth problem, with which we shall not be concerned here.

As soon as the concept was defined with precision, there arose the problem of studying topological properties of such group-manifolds. Indeed, in the first paper which systematically considers global Lie groups, H. Weyl's famous paper on linear representations [81], a key result which states that the fundamental group of a compact semisimple Lie group is finite is topological in nature. The question was next considered by E. Cartan in several papers, and later on by many mathematicians; as a matter of fact, it was often generalized in order to include also the study of "homogeneous spaces," i.e., manifolds which admit a transitive Lie group of homeomorphisms. A very complete survey of the work done in this field up to 1951 has been published in this Bulletin by H. Samelson [67]. Although of course some overlap is unavoidable, the present report is meant as a sequel and will therefore concentrate mainly on developments which occurred during these very last years. It will be devoted for the greater

An address delivered before the New York meeting of the Society on February 27, 1954 by invitation of the Committee to Select Hour Speakers for Eastern Sectional Meetings; received by the editors May 7, 1955.

1 This report also surveys material expounded at the Summer Mathematical Institute on Lie groups and Lie algebras, Colby College, 1953. The author expresses his hearty thanks to Dr. W. G. Lister, who prepared mimeographed notes of these lectures, which were helpful in the preparation of this paper.
\end{abstract}


part to homological properties of compact Lie groups, their classifying spaces and coset spaces. To complete the picture we also discuss at the end homotopy groups, noncompact Lie groups and their coset spaces and homogeneous complex spaces.

2. Outline of methods. There have been several lines of approach to the study of homological properties of compact Lie groups and coset spaces which, in first approximation, may be divided into three groups; methods of differential geometry, of algebraic topology, and the use of Morse theory.

The first direction was initiated by E. Cartan [16] who showed that the study of exterior differential forms on a coset space $G / U$ invariant under the operations of $G$ allows one to compute the Betti numbers of $G / U$ using de Rham's theorems (which were conjectured by Cartan for that purpose, and proved soon afterwards by de Rham). This method was applied to the classical groups by R. Brauer and to certain symmetric spaces by C. Ehresmann, and Iwamoto. Implicit in Cartan's construction was the notion of cohomology ring of a Lie algebra modulo a subalgebra which was explicitly formulated by Chevalley-Eilenberg [25] and which became Koszul's principal tool [41]. Under the influence of A. Weil this point of view was broadened and led to a theory of differentiable principal bundles which gave a framework to a modern exposition of $\mathrm{E}$. Cartan's theory of connections as well as new tools to study homology of coset spaces. The work of H. Cartan, C. Chevalley, J. L. Koszul and A. Weil in this direction has been summarized in $[20 ; 24 ; 42]$. Some of the main points are an algebraic transgression theorem in the Weil algebra (whose topological analog will be discussed in $\S 9$ ), a theorem of Chevalley connecting the Betti numbers of a Lie group and the invariants of its Weyl group (see \$9), a result of Cartan on homogeneous spaces (see §13), and a theory, due to Koszul, of a certain type of differential algebras. Besides its topological applications, the latter allows us to give a homological formulation of Hilbert's theory of syzygies; it was later on generalized by H. Cartan-S. Eilenberg (see their forthcoming book, Homological Algebra, Princeton Series, no. 19). This method makes full use of differential forms, and as far as topology is concerned, gives results only about real cohomology. Already discussed in [67], it will not be dealt with here anymore, since most of the results directly relevant to our subject have been obtained later on by topological methods, to which we now turn our attention. Roughly speaking, these and the results to which they lead may also be divided into three groups. 
The first group (see $\S \S 6,7)$ consists of general properties, "general" in the sense that they derive solely from the existence of a nontrivial product, say with unit, sometimes assumed to be associative. The interest of this approach was displayed first by H. Hopf [38] who showed that the real cohomology algebra of a compact connected manifold endowed with such a product is a Grassmann algebra. Subsequent work along these lines has been done by H. Samelson [66], J. Leray [45], and the author [2].

The second group uses fiber bundle theory and in particular Leray's spectral sequence. Its starting point is the existence of universal bundles (see $\S 8$ for definition) and is at the source of a great part of the recent progress in this field. It may be viewed as a study of relations between on one hand cohomological and group-theoretical properties of a compact Lie group $G$, and on the other hand universal properties of characteristic classes of bundles with structural group $G$. Thus it connects topology of Lie groups with a problem of fiber bundle theory, and the simultaneous consideration of these two questions has allowed us to gain new information on both. The main results are described in $\S \S 8,9$ and the most important examples are given in $\$ 10$; more or less direct applications to coset spaces and Lie groups are discussed in $\S \S 11$ to 14 .

The results obtained so far from these two points of view do not give a systematic and effective procedure to compute the cohomology over a field of characteristic not zero of a given coset space. In order to determine it (whenever possible), one has to use "special devices" taking advantage of some particular property of the space under consideration, and these make up the third group mentioned above. The main ones are cellular decompositions (see \$15) and the study of special fiberings combined with the general results on universal bundles; $\$ 11$ indicates the present state of knowledge concerning the cohomology of the compact simple Lie groups.

The use of Morse theory in these questions was recently initiated by $R$. Bott [13] who derives topological properties of certain coset spaces and of the space of loops on a Lie group by the study of geodesics on compact Lie groups. Their properties are obtained from the theory of singular elements, due to H. Weyl and E. Cartan, which is thus connected with topology in an entirely new way. The results announced in $[13 ; 14]$, will be mentioned in $\S \S 11,12,15,18$.

3. Algebra. It will be convenient to recall in $\S \S 3,4,5$ some known facts and definitions and to fix notations.

$p$ will denote either a prime number or zero, $Z_{p}$ ( $p$ prime) the field 
of integers $\bmod p, Z_{0}$ the field of rational numbers, $R$ the real numbers, $K$ an arbitrary field.

A module $M$ over a ring $A$ is graded if it is the direct sum of submodules to be denoted here by $M^{i}$ (sometimes $M_{i}$ ), the index running through the positive integers; the degree $d^{0} x$ of an element $x \neq 0$ is the smallest integer $i$ such that $x \in \sum_{j \leqq i} M^{i}$, and the elements of $M^{i}$ are the homogeneous elements of degree $i$.

A graded algebra over a ring $A$ is a graded module where $H^{i} \cdot H^{j}$ $\subset H^{i+i}$; the unit, if any, is then contained in $H^{0}$. The algebra, or the product, is said to be anticommutative if

$$
a \cdot b=(-1)^{i \cdot j} b \cdot a, \quad\left(a \in H^{i}, b \in H^{j}\right),
$$

when $A=Z_{2}$, this is just commutativity; if $A$ is a field of characteristic $\neq 2$, then every homogeneous element of odd degree has square zero.

The exterior algebra of a vector space $P$ over a field $K$ (or of a free abelian group) is denoted $\Lambda(P)$ or $\Lambda\left(x_{1}, \cdots, x_{s}\right)$ where the $x_{i}$ 's form a base of $P$; the latter will always be graded, and the $x_{i}$ be homogeneous; $\Lambda(P)$ is then a graded algebra under the exterior product. When $K$ has characteristic $\neq 2$, the exterior product is anticommutative if and only if the $x_{i}$ have odd degrees.

The elements $\left(x_{i}\right)$ of an algebra $H$ form a simple system of generators if $H$ is the weak direct sum of the $A$-modules generated by the unit, if any, and by the elements

$$
x_{i_{1}} \cdot x_{i_{2}} \cdots \cdot x_{i_{k}} \quad\left(i_{1}<i_{2}<\cdots<i_{k} ; 1 \leqq k \leqq s\right) .
$$

We write in this case $H=\Delta\left(x_{1}, \cdots, x_{s}\right)$. The additive basis is the same as for the exterior algebra $\wedge\left(x_{1}, \cdots, x_{s}\right)$, but the former differs in that no requirement is made about $x_{i} x_{j}+x_{j} x_{i}$.

As usual $A\left[x_{1}, \cdots, x_{s}\right]$ is the ring of polynomials in the indeterminates $x_{1}, \cdots, x_{s}$ with coefficients in the commutative ring $A$. We denote by $S\left(x_{1}, \cdots, x_{8}\right)$ the ring of symmetric polynomials in the $x_{i}$ 's and by $\sigma_{i}\left(x_{1}, \cdots, x_{s}\right)$ the $i$ th elementary symmetric function.

If $H_{1}$ and $H_{2}$ are two graded algebras over a ring $A, H_{1} \otimes H_{2}$ is their (skew-)-tensor product over $A$ : as a module, it is the usual tensor product, but the product is defined by

$$
(a \otimes b) \cdot(c \otimes d)=(-1)^{i j}(a \cdot c \otimes b \cdot d), \quad\left(b \in H_{2}^{i}, c \in H_{1}^{j}\right) ;
$$

it has three gradings, but the one we shall consider usually is the total degree defined by

$$
\left(H_{1} \otimes H_{2}\right)^{i}=\sum_{s+t=i} H_{1}^{s} \otimes H_{2}^{t} .
$$


If $H_{1}$ and $H_{2}$ are anticommutative, so is $H_{1} \otimes H_{2}$ with respect to the total degree.

4. Homology and cohomology groups. We shall not specify the homology and cohomology groups of a space $X$. Actually, since almost all the spaces considered, at least up to $\$ 16$, are compact differentiable manifolds and hence finite polyhedra, most of the time it would suffice to use simplicial homology and cohomology groups. $H^{i}(X, A)$ (resp. $\left.H_{i}(X, A)\right)$ is the $i$ th cohomology (resp. homology) group of $X$ with coefficients in the abelian group $A$, and $H^{*}(X, A)$ (resp. $\left.H_{*}(X, A)\right)$ is the direct sum of the $H^{i}(X, A)\left(\right.$ resp. $\left.H_{i}(X, A)\right)$. We shall in general consider cohomology so that, when $A$ is a ring, we have a product in $H^{*}(X, A)$, the so-called cup-product; it is associative, distributive, adds the degrees, has a unit, spanning $H^{0}(X, A)$ when $X$ is connected, and is anticommutative when $A$ is commutative.

The homology and cohomology groups depend on the coefficients, the most complete information being obtained when $A$ is the ring of integers $Z$. In this case we have ( $X$ being a finite polyhedron):

$$
H^{i}(X, Z)=F^{i}+T^{i}
$$

with $F^{i}$ free abelian and finitely generated, $T^{i}$ finite; the $T^{i}$ define the torsion of the space $X$. In particular we say that $X$ has $p$-torsion ( $p$ prime) if one of the $T^{i}$ has order divisible by $p$; to simplify certain statements we also allow $p$ to be zero and agree that a space is always without 0 -torsion. The study of integral cohomology groups is often difficult and it is convenient to first investigate the cohomology over various fields of coefficients choosing one for each characteristic. Roughly speaking, we may say that the knowledge of $H^{*}\left(X, Z_{0}\right)$ gives information on the $F^{i}$, but says nothing about torsion; $H^{*}\left(X, Z_{0}\right)$ and $H^{*}\left(X, Z_{p}\right)$ give rather precise, though not complete, information on the $p$-primary components of the torsion groups $T^{i}$.

In case of a field of coefficients the $H^{i}(X, K)$ and $H_{i}(X, K)$ are vector spaces, dual to each other. The dimension of $H^{i}\left(X, Z_{0}\right)$ (resp. $\left.H^{i}\left(X, Z_{p}\right)\right)$ is the $i$ th Betti number (resp. the $i$ th Betti number $\bmod p$ ) of $X$. Finally we denote by $P_{p}(X, t)$ the Poincaré polynomial

$$
P_{p}(X, t)=\sum_{i} \operatorname{dim} H^{i}\left(X, Z_{p}\right) \cdot t^{i}
$$

As is well known the Poincaré polynomial of a cartesian product $X \times Y$ is the product $P(X, t) \cdot P(Y, t)$, and more generally

$$
H^{*}(X \times Y, K)=H^{*}(X, K) \otimes H^{*}(Y, K)
$$


(and similarly for homology).

A continuous map $f: X \rightarrow Y$ induces a (degree preserving) homomorphism of $H^{*}(Y, A)$ (resp. $H_{*}(X, A)$ ) into $H^{*}(X, A)$ (resp. $\left.H_{*}(Y, A)\right)$ to be denoted by $f^{*}$ (resp. $f_{*}$ ).

5. Compact Lie groups. We denote by $G / U$ the space of left cosets of a Lie group $G$ modulo a closed subgroup $U$, endowed with the usual quotient topology. It is an analytic manifold on which $G$ acts transitively and analytically by means of left translations. Conversely any manifold on which a Lie group acts transitively is homeomorphic to a coset space $G / U$ by a homeomorphism which commutes with the operations of $G$.

A Lie group is compact, or connected, if its underlying manifold is compact or connected. Two Lie groups are locally isomorphic if there exists a homeomorphism between two neighborhoods of the identities compatible with the product.

Any abelian compact connected $n$-dimensional Lie group is isomorphic to a torus $T^{n}$, i.e., to the direct product of $n$ copies of the multiplicative group of complex numbers with norm 1. As is well known, the maximal tori of a compact Lie group $G$ are conjugate to each other by inner automorphisms; their common dimension is the rank of $G$; a maximal torus will be in general denoted by $T$, since the omission of the rank will not bring confusion. A maximal torus has finite index in its normalizer $N_{T}$ and the quotient $N_{T} / T$ is a finite group, the Weyl group of $G$, to be denoted by $W(G)$; it has an obvious representation as automorphism group of $T$, which is faithful when $G$ is connected.

A compact connected Lie group is simple if it has no proper closed invariant subgroups of strictly positive dimension, it is semi-simple if its center is finite. Compact connected Lie groups are locally isomorphic to (and in fact finitely covered by) direct products of tori and simple non-abelian groups, so that their classification reduces to that of simple groups. The different classes of locally isomorphic compact connected simple groups are usually denoted by the symbols $A_{r}(r \geqq 1), B_{r}(r \geqq 2), C_{r}(r \geqq 3), D_{r}(r \geqq 4)$ (the classical structures), and $G_{2}, F_{4}, E_{6}, E_{7}, E_{8}$ (the exceptional structures); the corresponding groups have dimension $r(r+2), r(2 r+1), r(2 r+1), r(2 r-1), 14,52$, $78,133,248$ respectively; the subscript denotes the rank. Each of these symbols may represent several groups, as will be discussed more thoroughly in $\$ 11$. For the moment, we simply recall that the classical structures are represented by well known linear groups, namely: 
$A_{r}$ by the group $S U(r+1)$ of $r+1 \times r+1$ complex unitary matrices of determinant +1 .

$B_{r}$ (resp. $D_{r}$ ) by the group $S O(2 r+1)$ (resp. $S O(2 r)$ ) or real orthogonal $2 r+1 \times 2 r+1$ (resp. $2 r \times 2 r$ ) matrices of determinant +1 , or by the spinor group $\operatorname{Spin}(2 r+1)$ (resp. Spin $(2 r))$.

$C_{r}$ by the group $S p(r)$ of $r \times r$ quaternionic unitary matrices. They are of course all defined for $r \geqq 1$, but the restriction on the indices was made above in order to get each structure exactly once. We recall that

$$
A_{1}=B_{1}=C_{1}, \quad B_{2}=C_{2}, \quad A_{3}=D_{3}, \quad D_{2}=A_{1} \times A_{1} .
$$

The full group of real orthogonal (resp. complex unitary) matrices will be denoted by $O(n)$ (resp. $U(n)$ ).

6. Hopf's and Samelson's theorems. An $H$-space is a space endowed with a binary continuous law of composition with unit (in fact a weaker condition is usually postulated, but this is of no importance here). Hopf's theorem recalled in $\$ 2$ admits the following generalization [2]: Let $X$ be a connected finite polyhedron which is an $H$-space. Then $H^{*}\left(X, Z_{p}\right)$ has a minimal system of homogeneous generators $\left(x_{i}\right),(1 \leqq i \leqq m)$, such that the monomials

$$
x_{1}^{r_{1}} \cdot x_{2}^{r_{2}} \cdots x_{m}^{r_{m}} \quad\left(0 \leqq r_{i}<s_{i}, x_{i}^{s_{i}-1} \neq 0, x_{i}^{s_{i}}=0\right)
$$

form an additive basis; moreover, for $p=0, d^{0} x_{i}$ is odd and $s_{i}=2$; for $p=2, s_{i}$ is a power of 2 ; for $p \neq 0,2, s_{i}=2$ when $d^{0} x_{i}$ is odd and $s_{i}$ is a power of $p$ when $d^{0} x_{i}$ is even. A suitable modification of that statement applies also to infinite dimensional $H$-spaces, but will not be considered here. A consequence of this theorem is that if $X$ has no $p$-torsion (resp. no torsion) its cohomology ring over $Z_{p}$ (resp. $Z$ ) is the exterior algebra of a subspace (resp. free abelian group) generated by elements of odd degrees. As in Hopf's case, this follows from a purely algebraic result. Let us say that an algebra $H$ over a field $K$ is a Hopf algebra if it is graded by subspaces $H^{i}(i \geqq 0)$, has a unit generating $H^{0}$, is associative, distributive, anticommutative, and if there is a homomorphism $h^{*}$ of $H$ into $H \otimes H$ such that for $x$ homogeneous and $\neq 0$ we have

$$
h^{*}(x)=x \otimes 1+1 \otimes x+\sum_{1}^{k} u_{i} \otimes v_{i} \quad\left(0<d^{0} u_{i}<d^{0} x=d^{0} u_{i}+d^{0} v_{i}\right) .
$$

If $X$ is an $H$-space, the product $h: X \times X \rightarrow X$ induces a map

$$
h^{*}: H^{*}\left(X, Z_{p}\right) \rightarrow H^{*}\left(X \times X, Z_{p}\right) \cong H^{*}\left(X, Z_{p}\right) \otimes H^{*}\left(X, Z_{p}\right)
$$


which satisfies the previous condition and the above theorem follows from the fact that every finite dimensional Hopf algebra over a perfect field has a system of generators $\left(x_{i}\right)$ with the above mentioned properties. Standard facts about binomial coefficients show that the unit and $x_{i}$ generate a subalgebra which is a Hopf algebra under the map $h_{b}^{*}$ defined by

$$
h_{i}^{*}\left(x_{i}\right)=x_{i} \otimes 1+1 \otimes x_{i} ;
$$

hence we may say that a Hopf algebra over a perfect field is isomorphic to the tensor product of Hopf algebras $H_{i}$ which are generated by one element (besides the unit). This does not mean however that the isomorphism carries $h^{*}$ over to the tensor product of the $h_{i}^{*}$. The existence of such an isomorphism is essentially equivalent to the existence of a system of generators which are "primitive" elements, i.e., elements for which

$$
h^{*}(x)=x \otimes 1+1 \otimes x,
$$

and examples show that this need not be the case. However, it is true in one important particular case: when $H$ is an exterior algebra generated by elements of odd degrees and when $h^{*}$ is "associative," i.e., when the two homomorphisms $h^{*} \otimes 1$ and $1 \otimes h^{*}$ of $H \otimes H$ into $(H \otimes H) \otimes H$ and $H \otimes(H \otimes H)$ become identical under the standard isomorphism of the two triple products. This is Samelson's theorem or more precisely the cohomological version of it (see also $\$ 7$ ) proved in characteristic zero by Samelson [66], and later on by J. Leray [45], whose argument is valid for arbitrary characteristic, and even for the exterior algebra of a free abelian group. The associativity condition is of course satisfied in the case of $H$-spaces with an associative product, in particular for Lie groups, for which Samelson's theorem can also be obtained via transgression theorems $[2, \S 20]$.

Even for cohomology algebras of Lie groups, the properties of $h^{*}$, which in fact are those of the Pontrjagin product discussed below, are not completely known; they seem to be closely connected with the universal spectral sequence (defined in \$9), witness e.g. the fact that a universally transgressive element is primitive $[2, \S 20]$. This, combined with the results of $\S 9$, shows that, very often, $H^{*}\left(G, Z_{p}\right)$ is generated by primitive elements, but not much is known about $h^{*}$ when this fails to be true, as e.g. in the case of $H^{*}\left(\operatorname{Spin}(n), Z_{2}\right)$ $(n \geqq 10)$ (see [5]).

We already pointed out that if the $H$-space $X$ (always supposed to be a finite polyhedron) has no torsion, then $H^{*}(X, Z)$ is the exterior algebra of a free abelian group graded by odd degrees, and 
spanned by primitive elements when the product is associative. More generally the same conclusion applies to the quotient $H^{*}(X)$ of $H^{*}(X, Z)$ by its torsion ideal. In fact, the Künneth rule shows first that $h^{*}$ induces a homomorphism of $H^{*}(X)$ into $H^{*}(X) \otimes H^{*}(X)$ satisfying Hopf's condition (6.1), then the generalized Hopf's theorem, applied to $H^{*}(X) \otimes Z_{p}$ for all $p$, gives the first statement and the second one follows from Leray's proof of Samelson's theorem (for compact Lie groups these facts are also proved in [29]). It follows that for $p \neq 2, H^{*}\left(X, Z_{p}\right)$ contains an exterior subalgebra having the same Poincaré polynomial as $H^{*}\left(X, Z_{0}\right)$; it would have some interest to know more about this embedding.

In the general case, nothing is known about the cohomology ring of an $H$-space over the integers or over a ring (say the integers modulo a power of a prime) which is not a field. A chief difficulty is of course that $H^{*}(X \times X, A)$ is not the tensor product of $H^{*}(X, A)$ with itself, but an extension of it by the Tor-product of $H^{*}(X, A)$ with itself.

7. The Pontrjagin product. Again let $X$ be a connected $H$-space, with associative product $h$. The latter defines a map $h_{*}$ of $H_{*}(X \times X, K)$, which we may identify with $H_{*}(X, K) \otimes H_{*}(X, K)$, into $H_{*}(X, K)$, and $h_{*}(a \otimes b)$ is called the Pontrjagin product of $a$ and $b$ : thus $H_{*}(X, K)$ becomes a ring under a product, first considered by Pontrjagin [62], which is associative, distributive, adds the degrees, has a unit spanning $H_{0}(X, K)$, but, unlike the cup-product, is not necessarily anticommutative, and that last fact makes its study more difficult. It may be remarked that the ring $H_{*}(X, K)$ together with the map $d_{*}$ induced by the diagonal map $d: x \rightarrow(x, x)$ of $X$ into $X \times X$ satisfies all conditions imposed on a Hopf algebra except for anticommutativity, but there is no structure theorem analogous to the generalized Hopf theorem of $\$ 6$; in fact Bott-Samelson (Comment. Math. Helv. vol. 27 (1954) pp. 320-337) have given examples of infinite dimensional $H$-spaces whose homology rings are free associative algebras. Even for Lie groups the Pontrjagin product is not always anticommutative, as the following example shows [5]: $H_{*}\left(\right.$ Spin $\left.(10), Z_{2}\right)$ has a simple system of generators $x_{3}, x_{5}, x_{6}, x_{7}, x_{9}$, $x_{15}\left(d^{0} x_{i}=i\right)$, with the following relations:

$$
\begin{aligned}
& x_{i} \cdot x_{i}=0(\text { all } i), \quad x_{i} x_{j}=x_{j} \cdot x_{i} \quad(i<j,(i, j) \neq(6,9)), \\
& x_{6} \cdot x_{9}=x_{9} \cdot x_{6}+x_{15} .
\end{aligned}
$$

This is so far the only case in which the homology ring of a Lie group over a field is completely known and is not an anticommutative ex- 
terior algebra; its structure is still reasonably simple and it does not seem too unlikely that homology rings of finite-dimensional $\mathrm{H}$-spaces have more common properties than have been found so far.

The homomorphisms $h^{*}$ and $h_{*}$ are transposes of each other under the standard duality between homology and cohomology over a field; hence $h^{*}$ and the Pontrjagin product determine each other. It is also easily seen that if $H^{*}(X, K)$ has a simple system of primitive generators $\left(x_{i}\right)(1 \leqq i \leqq m)$, then

$$
H_{*}(X, K)=\wedge\left(y_{1}, \cdots, y_{m}\right) \quad\left(d^{0} y_{i}=d^{0} x_{i}, i=1, \cdots, m\right),
$$

and conversely. By Samelson's theorem, the assumption is fulfilled in characteristic zero (and in fact the conclusion is Samelson's formulation of this result), and also in characteristic $p$ when $H^{*}\left(X, Z_{p}\right)$ is an exterior algebra generated by elements of odd degrees.

The Pontrjagin product admits a useful generalization. Let $M$ be a space on which an $H$-space $X$ operates, i.e. we have a continuous map $g: M \times X \rightarrow X$ such that

$$
\begin{aligned}
g(m, e) & =m \quad(e \text { the identity element }), \\
g(m, x \cdot y) & =g(g(m, x), y) .
\end{aligned}
$$

There is induced a map $g_{*}$ of $H_{*}(M \times X, K)$, which is identified with $H_{*}(M, K) \otimes H_{*}(X, K)$, into $H_{*}(M, K)$, and the image of $a \otimes b$ $\left(a \in H_{*}(M, K), b \in H_{*}(X, K)\right)$ will also be called the Pontrjagin product of $a$ and $b$. By duality, one also gets a pairing of $H^{*}(M, K)$ and $H^{*}(X, K)$ to $H^{*}(M, K)$. If $X$ operates on another space $N$ and if there is a continuous map $f: M \rightarrow N$ commuting with the operations of $X$, then $f^{*}$ commutes with the Pontrjagin product in the obvious way. More generally, one may define a pairing of the spectral sequence $\left(E_{r}\right)$ of $f$ and of $H_{*}(X, K)$ to $\left(E_{r}\right)$. When $X$ is a Lie group, this was considered by J. Leray $[46 ; 47]$ and later on, for fiber maps, by $T$. Kudo $[44]$ and the author $[2 ; 5]$. An analogous situation, involving spaces of loops, is the subject of Bott-Samelson's paper already mentioned. This pairing allows one e.g. to prove the following generalization of some results of $[2 ; 47 ; 66]$, (see $[5, \S 3])$ : Let $G$ be a compact connected Lie group operating on a space $M$ and let $f$ be a map of $G$ into $M$ commuting with the operations of $G$ on $M$ and onto itself by left translations. Assume $H^{*}\left(G, Z_{p}\right)$ to be an exterior algebra generated by elements of odd degrees. Then the image of $f^{*}$ is generated by primitive elements, and if $p \neq 2, H^{*}\left(M, Z_{p}\right)=A \otimes B$ where $f^{*}$ is $1-1$ on $A$ and annihilates the elements of strictly positive degrees in $B$. 
8. Characteristic classes, principal and universal bundles. For more details about the facts of fiber bundle theory discussed in the sequel, we refer once and for all to $[19 ; 48 ; 73]$.

The notion of characteristic class arose in 1935 in the work of Stiefel and Whitney about vector fields on manifolds. It is well known that on a compact manifold $M$ it is not always possible to construct a field of nonzero tangent vectors, depending continuously on their origin. However, this can be done up to a finite number of points and to each of these points there corresponds a certain integer, the "index of singularity" of the vector field at the point. By a well known result of H. Hopf the sum of these indices is equal to the Euler-Poincaré characteristic of $M$ (in our notations to $P_{0}(M,-1)$ ); in particular it is independent of the vector field. Stiefel and Whitney studied a generalization of the problem: the existence of $k$ tangent vector fields, linearly independent at every point. To construct them, one starts from a triangulation of $M$, defines the vector fields arbitrarily at the vertices, then tries to extend the definition continuously to the edges, 2-dimensional faces, and so on. This is always possible up to the $(n-k)$-dimensional simplices of the triangulation, but not necessarily to the $(n-k+1)$-dimensional skeleton. Thus we are led to consider the "indices of singularity" attached to the $(n-k+1)$-dimensional simplices; to their sum there corresponds an $(n-k+1)$-dimensional cohomology class $w_{n-k+1}$ which is called the $(n-k+1)$ st StiefelWhitney class of $M$. The important point is that it depends only on $M$, and not on the construction of the vector fields. We shall omit discussion of the natural coefficient systems with respect to which $w_{i}$ is taken, and for simplicity consider $w_{i}$ to be an element of $H^{i}\left(M, Z_{2}\right)$. Let us just mention that when $M$ is orientable and oriented, $w_{n}$ is in reality an integral class, equal by Hopf's theorem to the fundamental class multiplied by the Euler-Poincaré characteristic.

In 1942 Pontrjagin attached to a compact oriented manifold $M$ integral cohomology classes by a seemingly quite different procedure. He started from an embedding of $M$ into some euclidian space $E^{N}$ ( $N$ large), and by assigning to each point $P \in M$ the $n$-dimensional subspace parallel to the tangent space to $M$ at $P$, he defined a map $f$ of $M$ into the Grassmann manifold $G_{N, n}^{0}$ of oriented $n$-dimensional subspaces of $E^{N}$. By a careful study of its cohomology, he singled out certain elements $p_{i} \in H^{4 i}\left(G_{N, n}^{0}, Z\right)(1 \leqq 4 i \leqq n)$ which appeared to be basic and considered their images $f^{*}\left(p_{i}\right)$. These turned out to be independent of the embedding and therefore to be intrinsically attached to the given differentiable manifold. Subsequently, it was also shown that the Stiefel-Whitney classes can be defined in a sim- 
ilar fashion and, conversely, that the Pontrjagin classes are connected with certain problems on vector fields. Later on, S. S. Chern defined for a compact complex analytic manifold $M$ of complex dimension $n$, classes $C_{i} \in H^{2 i}(M, Z)(0 \leqq i \leqq n)$ which may be characterised either as "obstructions" to the construction of complex vector fields or as images of certain elements in the cohomology of the complex Grassmann manifold $H_{N, n}$ of $n$-dimensional subspaces of the complex affine space $C^{N}$ ( $N$ large), relative to a suitable map of $M$ into $H_{N, n}$.

A first generalization of these ideas depends on the notion of principal bundle. A space $E$ is a principal bundle with structural group $G$ (or as we shall say, a $G$-bundle), if $E$ is a transformation space for $G$ and if no transformation other than the identity has a fixed point (we assume $G$ to be compact, otherwise the definition is slightly more complicated). Then the orbits of $G$ are all homeomorphic to $G$ and are called the fibers. The space of orbits $B$ and the map of $E$ onto $B$ associating with each point in $E$ its orbit are called the base space and the projection. Examples: (1) Let $E$ be a topological group containing $G$ as a closed subgroup, and let $G$ operate on $E$ by right multiplication. The fibers are then the left cosets $x G$ and $B$ is the space of left cosets, denoted by $E / G$. (2) $B=M_{n}$ is an $n$-dimensional Riemannian manifold, $E$ the set of all orthonormal frames on $M_{n}$ (i.e. a point of $E$ is an orthonormal basis of the tangent space at some point of $M_{n}$ ), with a suitable topology. The orthogonal group $O(n)$ operates on $E$ in a natural way and $E$ becomes an $O(n)$-bundle: the fibers are the frames with a given origin and the projection assigns to each frame its origin. Similarly, if $M_{n}$ is orientable, the space of orthonormal frames corresponding to a given orientation is an $S O(n)$-bundle, and if $M$ is a complex analytic hermitian manifold, the space of unitary orthonormal frames on $M$ is a $U(n)$-bundle.

It next became apparent that the above characteristic classes may be associated with bundles of frames and moreover that the StiefelWhitney, Pontrjagin, and Chern classes may be defined for any $O(n)$, $S O(n)$, and $U(n)$-bundle respectively.

The final step in the generalization arose from the observation that the Grassmann manifolds were not necessary as "reference" spaces but could be replaced by what we now call classifying spaces. This generalization is valid for any compact Lie group $G$.

A principal bundle with structural group $G$ is said to be $n$-universal (for $G$ ) if its homology (or homotopy) groups vanish up to $n$ (except for $H_{0}$ of course). Such spaces exist for arbitrary compact Lie groups and any $n$, including $n=\infty$; we shall hereafter omit mentioning $n$. The base space $B_{G}$ of the universal bundle $E_{G}$ for $G$ is called a classify- 
ing space for $G$; its importance lies in the classification theorem which, roughly, states that the essentially different $G$-bundle structures with a given base $B$ are in 1-1 correspondence with the homotopy classes of maps $f: B \rightarrow B_{G}$. Therefore, to any $G$-bundle over $B$ there is attached a homomorphism $f^{*}$ of $H^{*}\left(B_{G}, A\right)$ into $H^{*}(B, A)$, the characteristic map of the fibering, the image of which is called the characteristic ring of the fibering. The characteristic classes of Stiefel-Whitney, Pontrjagin, and Chern appear then as images of particular elements in $H^{*}\left(B_{O_{(n)}}, Z_{2}\right), H^{*}\left(B_{S O(n)}, Z\right)$, and $H^{*}\left(B_{U(n)}, Z\right)$ respectively. Thus $H^{*}\left(B_{G}, A\right)$ may be viewed as the ring of "universal" characteristic classes for $G$-bundles; its properties are universal in the sense that, by the classification theorem, they are valid in any $G$-bundle, and are therefore quite important. More generally there is the problem of investigating the relations between the cohomology rings of $B_{G}$ and of $G$, in other words of the base and the fiber in the universal bundle $E_{G}$; this is precisely the problem alluded to in $\$ 2$; it will be discussed in $\$ 9$. In the study of this problem, an important role is played by a map $\rho(U, G)$ defined as follows. Let $U$ be a closed subgroup of $G$. Then $E_{G}$ is clearly a principal bundle with structural group $U$, with vanishing homotopy groups; hence it is also a universal bundle for $U$. The natural projection of $E_{G} / U$ onto $E_{G} / G$ may then be considered as a map of $B_{U}$ into $B_{G}$, to be denoted by $\rho(U, G)$.

Without entering into details, let us just mention in passing that $\rho(U, G)$ is a fiber map of a fibering of $B_{U}$, with typical fiber $G / U$, base $B_{G}$, and that $\rho(U, G)$ also occurs in the problem of restricting the structural group of a fiber bundle: the structural group of a bundle with base $B$ defined by a map $f: B \rightarrow B_{G}$ can be restricted to $U$ if and only if there exists a map $g: B \rightarrow B_{U}$ such that $f=\rho(U, G) \cdot g$.

9. Results on universal bundles. Invariants of the Weyl group. To express these results, we shall use the notion of transgression in a fiber bundle, and first recall briefly one of its possible definitions (see $[2, \S 5]$ for more details). Let $E$ be a bundle with fiber $F$, base $B$, projection map $\pi$ (e.g. a principal bundle, the only case in which this definition will be used below). Let $\pi^{\prime}$ (resp. $i^{\prime}$ ) be the map of cochains induced by $\pi$ (resp. the inclusion of a fiber in $E$ ). An element $x \in H^{s}(F, A)$ is transgressive in $E$ if there exists a cochain $c$ on $E$ such that $i^{\prime}(c)$ is a cocycle of $x$ and that its coboundary is of the form $\pi^{\prime}(b)$, where $b$ is some cochain on $B$, necessarily a cocycle. Its cohomology class $y \in H^{s+1}(B, A)$ is determined by $x$ only modulo a certain subgroup $L^{s+1}$ and the transgression is the map of the transgressive elements of $H^{s}(F, A)$ into $H^{s+1}(B, A) / L^{s+1}$ derived from 
$x \rightarrow y$. In spite of the fact that $y$ is not uniquely determined by $x$ in general, we shall write $y=\tau(x)$ whenever $y$ is obtained from $x$ by this procedure.

Let now $G$ be a compact connected Lie group. Then $x \in H^{s}(G, A)$ is universally transgressive if it is transgressive in $E_{G}$. The classification theorem shows that it is then transgressive in all $G$-bundles. The notion of transgression is interesting for at least two reasons: First, as we shall see, it is intimately related with the characteristic classes, and second a knowledge of the transgressive elements is quite useful in the computation of spectral sequences; this more technical point will not be illustrated here any further and we refer to $[2 ; 5]$ for examples.

The study of the homological properties of the universal bundle, i.e., essentially of its spectral sequence, the universal spectral sequence for $G$, appears thus to be a basic problem. So far, only partial results are known, the main one being:

(A) If $H^{*}\left(G, Z_{p}\right)$ is the exterior algebra of a subspace graded by odd degrees, then $H^{*}\left(G, Z_{p}\right)=\wedge\left(x_{1}, \cdots, x_{m}\right)$, with $x_{i}$ universally transgressive, of odd degree, and $H^{*}\left(B_{G}, Z_{p}\right)=Z_{p}\left[y_{1}, \cdots, y_{m}\right]$ with $y_{i}=\tau\left(x_{i}\right)(1 \leqq i \leqq m)$. Conversely if $H^{*}\left(B_{G}, Z_{p}\right)=Z_{p}\left[y_{1}, \cdots, y_{m}\right]$ with the $y_{i}$ 's of even degrees, then $H^{*}\left(G, Z_{p}\right)=\wedge\left(x_{1}, \cdots, x_{m}\right)$ with the $x_{i}$ 's universally transgressive and $y_{i}=\tau\left(x_{i}\right)(1 \leqq i \leqq m)$.

A similar result is valid over integers, when $G$ has no torsion (see $[2$, Théorème $19.1 ; 5$, Théorème 6.1$]$ ). The assumption of $(\mathrm{A})$ is fulfilled when $G$ has no $p$-torsion, and in particular in characteristic zero. An analogous, but weaker, result is:

(B) If $H^{*}\left(G, Z_{2}\right)$ has a simple system $\left(x_{i}\right)$ of universally transgressive generators, then $H^{*}\left(B_{G}, Z_{2}\right)=Z_{2}\left[y_{1}, \cdots, y_{m}\right]$, where $y_{i}=\tau\left(x_{i}\right)$ $(1 \leqq i \leqq m)$ and conversely.

(See [2, Proposition $19.1 ; 5$, Théorème 6.1]). In (B) we assume the existence of transgressive generators, whereas it is a part of the conclusion in (A); this assumption is often fulfilled, even when there is 2-torsion, e.g. in the case of $S O(n)(n \geqq 3), G_{2}, F_{4}$, Spin $(n)(7 \leqq n$ $\leqq 9)$. However it is not true for $H^{*}\left(\operatorname{Spin}(n), Z_{2}\right)$ when $n \geqq 10$ (see [5]).

Nothing is known up to now about $H^{*}\left(B_{G}, Z_{p}\right)$ outside the cases covered by (A) and (B); it seems that one has to expect quite different properties and that their study will require more knowledge about the behavior of reduced powers in the spectral sequence.

Let now $T$ be a maximal torus of $G$. Then

$$
H^{*}(T, Z)=\wedge\left(u_{1}, \cdots, u_{r}\right) \quad\left(d^{0} u_{i}=1, r=\text { dimension of } T\right),
$$


and by (A), or also by more direct considerations,

$$
H^{*}\left(B_{T}, Z\right)=Z\left[v_{1}, \cdots, v_{m}\right] \quad\left(v_{i}=\tau\left(u_{i}\right),(1 \leqq i \leqq r)\right) .
$$

The Weyl group $W(G)$ of $G$ operates on $T$, hence on its cohomology, and also on $H^{*}\left(B_{T}, Z\right)$, as is easily seen; we denote by $I_{G}$ the ring of polynomials in the latter invariant under $W(G)$; it is a direct summand, and $I_{G} \otimes Z_{p}$ is thus canonically embedded in $H^{*}\left(B_{T}, Z\right) \otimes Z_{p}$, which is just $H^{*}\left(B_{T}, Z_{p}\right)$, since $B_{T}$ has no torsion; it is of course contained in the ring of invariants of $W(G)$ operating on $H^{*}\left(B_{T}, Z_{p}\right)$, and is equal to it when $p=0$, but may be different from it otherwise. If we consider real cohomology, then we may identify $H^{*}\left(B_{T}, R\right)$ with the ring of polynomials over the Lie algebra of $T$, the operations of $W(G)$ being the obvious ones, but we have of course to give the "dimension" 2 to the coordinates $v_{i}$ when we consider them as elements of $H^{*}\left(B_{T}, R\right)$. The invariants of the Weyl group and the cohomology of $B_{G}$ are connected by the following theorem:

(C) Let $T$ be a maximal torus of a compact connected Lie group $G$. Assume $H^{*}\left(G, Z_{p}\right)$ to be the exterior algebra of an s-dimensional subspace graded by odd degrees. Then $s=\operatorname{dim} T$ and $\rho^{*}(T, G)$ maps $H^{*}\left(B_{G}, Z_{p}\right)$ isomorphically onto $I_{G} \otimes Z_{p}$.

A similar result is valid over integers when $G$ has no torsion. This theorem is established in $[2, \S \S 26,27]$, under the apparently slightly stronger (though in fact equivalent) assumption that $G$ has no $p$ torsion, but the proof is the same. Also, [2] assumes that $G / T$ has no $p$-torsion (resp. no torsion), a fact which has since been proved to be always true (see $\$ 15$ ). Theorems (A) and (C) have assumptions always fulfilled in characteristic zero and show therefore that the ring of invariants of the Weyl group has $r$ algebraically independent generators of (cohomological) dimensions $2 m_{1}, \cdots, 2 m_{r}$, where $H^{*}(G, R)=\wedge\left(x_{1}, \cdots, x_{r}\right) \quad\left(d^{0} x_{i}=2 m_{i}-1, i=1, \cdots, r\right)$, a result first obtained by C. Chevalley [24] $(r=\operatorname{rank} G)$.

Let $U$ be a closed connected subgroup of $G$, and $S \subset T$ be maximal tori of $U$ and $G$. When (A) can be applied to $U$ and $G$, the study of the homological position of $U$ in $G$, i.e., of the map $H^{*}(G, K) \rightarrow$ $H^{*}(U, K)$ induced by the inclusion, is essentially equivalent to that of $\rho^{*}(U, G)$. If moreover (C) can be used, then $\rho^{*}(U, G)$ is determined by the behavior of the invariants of $W(G)$ under the map $\rho^{*}(S, T)$, which in turn is explicitly described by means of the homomorphism $H^{1}(T, K) \rightarrow H^{1}(S, K)$ defined by the inclusion $S \subset T$ (see $[2, \S \S 28$ and 31$]$ for some applications). If $G=U(n), S p(n), S O(n)$, then the knowledge of $\rho^{*}(S, T)$ boils down to that of the weights (in E. Cartan's sense) of the linear group $U$. Thus we are led to connec- 
tions between weights and homological position of subgroups of the classical linear groups. For real cohomology, and starting from a point of view more akin to $[20 ; 24]$, they have been studied by E. B. Dynkin $[27 ; 28 ; 30 ; 31 \mathrm{a}]$.

An analogous, though different, example of relations between classifying spaces and group theoretical properties is offered in cohomology mod 2 by $O(n)$. Here we take instead of a maximal torus the subgroup $Q(n)$ of diagonal matrices which is isomorphic to $\left(Z_{2}\right)^{n}$; by a standard result

$$
H^{*}\left(B_{Q(n)}, Z_{2}\right)=Z_{2}\left[v_{1}, \cdots, v_{n}\right] \quad\left(d^{0} v_{i}=1 ; i=1, \cdots, n\right) .
$$

The quotient by $Q(n)$ of the normalizer of $Q(n)$ in $O(n)$ is readily seen to operate on $H^{*}\left(B_{Q(n)}, Z_{2}\right)$ as the full group of permutations of the $v_{i}$ 's. Then, in analogy with $(\mathrm{C})$, it may be shown that $\rho^{*}(Q(n), O(n))$ maps $H^{*}\left(B_{O_{(n)}}, Z_{2}\right)$ isomorphically onto the ring of symmetric functions in the $v_{i}$ 's, i.e., onto the ring of invariants of the "Weyl group" defined by using $Q(n)$ instead of $T$. Moreover, $\rho^{*}(Q(n), O(n))$ maps the $i$ th universal Stiefel-Whitney class $w_{i}$ (introduced in $\$ 8$ ) onto the $i$ th elementary symmetric function in the $v_{i}$ 's [3, Theorème 6.1]. A similar result is valid for the special orthogonal group.

Here again, not much is known outside these two cases. The latter one suggests the substitution of maximal abelian subgroups of type $(p, \cdots, p)$ for maximal tori when dealing $\bmod p$ with a group having $p$-torsion, but examples show that one cannot expect relations as simple as for $O(n)$ in general, though on the other hand these subgroups seem definitely to be related to the $p$-torsion of the group (see $\$ 12)$.

10. Examples. We describe here briefly some relations between the results of $\S 9$ and the characteristic classes introduced in $\S 8$, using some facts on the cohomology of the classical groups (see $\$ 11$ for references), and on their structure (see e.g. [74]).

$U(n)$ has rank $n$ and no torsion. The Weyl group operating on $H^{*}\left(B_{T}, Z\right)=Z\left[v_{1}, \cdots, v_{n}\right]$ is the group of permutations of the $v_{i}$ 's, and $\rho^{*}(T, U(n))$ maps $H^{*}\left(B_{U(n)}, Z\right)$ isomorphically onto the ring $S\left(v_{1}, \cdots, v_{n}\right)$ of symmetric functions. The universal $i$ th Chern class is mapped on the $i$ th elementary symmetric function. Thus $H^{*}\left(B_{U(n)}, Z\right)$ is the ring of polynomials in the Chern classes, and moreover $H^{*}(U(n), Z)=\bigwedge\left(x_{1}, \cdots, x_{n}\right)$, where $C_{i}=\tau\left(x_{i}\right)$ and therefore $d^{0} x_{i}=2 i-1$.

The unitary symplectic group $S p(n)$ has rank $n$ and no torsion. $W(S p(n))$ is the group of permutations of the $v_{i}$ combined with 
arbitrary changes of signs; hence $I_{S p(n)}=S\left(v_{1}^{2}, \cdots, v_{n}^{2}\right)$ : the ring $H^{*}\left(B_{S p(n)}, Z\right)$ is generated by elements of dimensions $4 i(1 \leqq i \leqq n)$, and $H^{*}(S p(n), Z)$ by elements of dimensions $4 i-1(1 \leqq i \leqq n)$.

$S O(2 n+1)$ (resp. $S O(2 n))$, has rank $n$ and its Weyl group consists of the permutations of the $v_{i}$ 's together with an arbitrary (resp. even) number of changes of signs. Hence $I_{S O(2 n+1)}=S\left(v_{1}^{2}, \cdots, v_{n}^{2}\right)$ and $I_{S O(2 n)}$ is generated by $S\left(v_{1}^{2}, \cdots, v_{n}^{2}\right)$ and the product $v_{1} \cdots v_{n}$. For $p \neq 2, S O(m)$ has no $p$-torsion $(m=1,2,3, \cdots)$ and we may apply (A) and (C). Also, it turns out that $\rho^{*}(T, S O(m))$ maps the $i$ th universal Pontrjagin class, reduced $\bmod p$, onto $\sigma_{i}\left(v_{1}^{2}, \cdots, v_{n}^{2}\right)$. In fact, this is also true over the integers, though in that case the kernel of $\rho^{*}(T, S O(m))$ is not zero. In the case $m=2 n$, the product $v_{1} \cdots v_{n}$ plays a special role, too; it is the image under $\rho^{*}(T, S O(2 n))$ of the so called Euler-Poincare class $W_{m}$. The latter is the unique element of $H^{2 n}\left(B_{S O(2 n)}, Z\right)$ having the following property: if $M$ is a $2 n$-dimensional orientable compact oriented manifold, and if $f: M \rightarrow B_{S O(2 n)}$ is the map corresponding to the bundle of orthonormal frames (see §8), then $f^{*}\left(W_{m}\right)$ is equal to the generator of $H^{2 n}(M, Z)$ singled out by the orientation, multiplied by the Euler-Poincaré characteristic of $M$.

Analogously, it may be shown that for $m=2 n, 2 n+1$, and for $p \neq 2, \quad \rho^{*}(T, \quad O(m))$ maps $H^{*}\left(B_{O_{(m)}}, \quad Z_{p}\right)$ isomorphically onto $S\left(v_{1}^{2}, \cdots, v_{n}^{2}\right)$. One can also introduce a universal Pontrjagin class $P_{i} \in H^{4 i}\left(B_{O(m)}, Z\right)$, (i.e., for $O(m)$ bundles and not as before for $S O(m)$ bundles $)$; it is mapped onto $\sigma_{i}\left(v_{1}^{2}, \cdots, v_{n}^{2}\right)$ by $\rho^{*}(T, O(m))$ (also over integers).

The cohomology mod 2 and the relations with Stiefel-Whitney classes have already been mentioned in $\$ 9$. A closer investigation shows that in $H^{*}\left(B_{O_{(n)}}, Z\right)$ and $H^{*}\left(B_{S O_{(n)}}, Z\right)$ all torsion elements have order 2 . This implies that an element of the integral cohomology ring is completely determined by its reductions mod 2 and over the rationals. The $i$ th Pontrjagin class may then be characterised as the element which is mapped on to the $i$ th elementary symmetric function in the $v_{i}$ 's in $H^{*}\left(B_{T}, Z_{0}\right)$ and onto the square of the $2 i$ th elementary symmetric function in $H^{*}\left(B_{Q(n)}, Z_{2}\right)$.

Some of the above results for the orthogonal groups are not in the literature, but follow without difficulty from known facts. A complete discussion may be found in mimeographed notes of lectures held by the author at the University of Chicago, Fall 1954.

The interpretation of characteristic classes as elementary functions gives immediate interpretations or proofs of their so-called Whitney duality properties. It also leads to a method of computation of their Steenrod reduced powers and, via transgression, of reduced powers in 
the classical Lie groups. This in turn has applications to problems of differential geometry like existence of vector fields or of almost complex structures on spheres (see [12]).

11. Homology and cohomology of compact simple Lie groups. The different classes of locally isomorphic compact simple Lie groups have been listed in $\$ 5$. Each of them contains a simply connected representative, unique up to global isomorphism, and the other groups are quotients of the former by subgroups of its center.

For $A_{r}, B_{r}, C_{r}$ the simply connected groups are

$$
S U(r+1), \quad \operatorname{Spin}(2 r+1), \quad S p(r)
$$

whose centers are cyclic of orders $r+1,2,2$. The quotients by the full centers are the groups $P U(r+1), S O(2 r+1), P S p(r)$ of projective transformations induced in the complex $r$-dimensional, real $2 r$ dimensional, quaternionic $(r-1)$-dimensional projective space respectively.

The simply connected group of structure $D_{r}$ is Spin (2r), its center is of order 4, cyclic if $r$ is odd, noncyclic otherwise; the quotient of order 4 is the projective orthogonal group $P S O(2 r)$. For $r$ odd, there is one quotient of order 2, which is $S O(2 r)$; for $r$ even there are besides $S O(2 r)$ the two "semi-spinor" groups. The latter are homeomorphic to each other for all $r$, and in particular for $r=4$, are isomorphic to $S O(8)$ by the triality principle; however, it is not known to the author whether the semi-spinor group is homeomorphic to the corresponding orthogonal group in general.

Finally the simply connected representatives of the structures $G_{2}, F_{4}, E_{6}, E_{7}, E_{8}$ have cyclic centers of orders $1,1,3,2$, 1. (For all this, see e.g. E. Cartan, Annali di Matematica vol. 4 (1927) pp. 209256).

Up to now, the following information on the homological properties of these groups has been obtained.

(11.1) The real cohomology for all groups (see e.g. [2], [24], [66], and [67] for other references). Two locally isomorphic groups have the same real cohomology (see e.g. [62], it also follows from standard theorems on coverings, or from statement (C) in $\S 9$ ); hence it is a property of a class of locally isomorphic groups. For $E_{6}, E_{7}, E_{8}$ the degrees of the primitive generators are $(3,9,11,15,17,23),(3,11$, $15,19,23,27,35),(3,15,23,27,35,39,47,59)$. For the other structures, see below.

(11.2) The integral cohomology ring of $S U(n), S p(n), G_{2}, F_{4}$. The groups $S U(n)$ and $S p(n)$ have no torsion $[36 ; 62 ; 2]$ and 


$$
\begin{aligned}
H^{*}(S U(n), Z) & =\wedge\left(x_{3}, x_{5}, \cdots, x_{2 n-1}\right), \\
H^{*}(S p(n), Z) & =\wedge\left(x_{3}, x_{7}, \cdots, x_{4 n-1}\right), \quad\left(d^{0} x_{i}=i\right) .
\end{aligned}
$$

$G_{2}$ has 2 -torsion and $H^{*}\left(G_{2}, Z\right)$ has 2 generators $h_{3}, h_{11}$ of degrees 3,11 such that $h_{3}^{4}=h_{11}^{2}=0$ and that $H^{*}\left(G_{2}, Z\right)$ is the weak direct sum of the infinite cyclic groups generated by $1, h_{3}, h_{11}, h_{3} \cdot h_{11}$ and of the groups of order 2 generated by $h_{3}^{2}, h_{3}^{3}$ (see $[5, \S 17]$ ).

$F_{4}$ has torsion coefficients of order $2,3,6$, and $H^{*}\left(F_{4}, Z\right)$ is isomorphic to the product

$$
U \otimes H^{*}\left(G_{2}, Z\right) \otimes \wedge(x) \quad\left(d^{0} x=15\right),
$$

where $U$ is a graded algebra with unit defined by

$$
U^{0}=U^{23}=Z, \quad U^{8}=U^{8} \cdot U^{8}=U^{16}=Z_{3}, \quad U^{i}=0 \text { otherwise }
$$

(see $[5, \S 23])$.

(11.3) Spin $(n)(n \leqq 6)$ has no torsion; $S O(n)(n \geqq 3)$ and Spin $(n)$ $(n \geqq 7)$ have 2-torsion and all their torsion coefficients are equal to 2 (see $[2 ; 35 ; 56 ; 62]$ for $S O(n)$, and $[5]$ for Spin $(n))$. Hence their integral cohomology rings are theoretically determined by their cohomology rings over $Z_{0}$ and $Z_{2}$; however the explicit formulas seem to be quite cumbersome and have not been written down.

Added in proof. Other proofs of the results about $\operatorname{Spin}(n)$ mentioned in (11.3), (11.4) have been recently given by S. Araki (Memoirs of the Faculty of Science. Kyusyu Imperial University, Series A, vol. 9 (1955) pp. 1-35). He uses a cellular decomposition which is a covering of the J. H. C. Whitehead cellular decomposition of $S O(n)$, the basic tool of Miller [56].

(11.4) The cohomology $\bmod p($ all $p)$ for $G_{2}, F_{4}$ and all the groups of the classical structures (with the possible exception of the semispinor groups of type $D_{2 r}(r \neq 2)$ in cohomology mod 2), with partial or complete information on Steenrod's reduced powers. In the case of $G_{2}, F_{4}, S U(n), S p(n)$ the cohomology ring can be read off (11.2). For the reduced powers see $[5 ; 12]$; for the $S q^{i}$ in $S O(n)$, see $[3 ; 56]$. Moreover, $H^{*}\left(G_{2}, Z_{2}\right)$ and $H^{*}\left(F_{4}, Z_{2}\right)$ have simple systems of universally transgressive generators of degrees $(3,5,6)$ and $(3,5,6,15$, 23) respectively.

Let $s$ be the greatest power of 2 dividing a given integer $n$. Then $H^{*}\left(P S p(n), Z_{2}\right)$

$$
=Z_{2}[a] /\left(a^{4 s}\right) \otimes \wedge\left(x_{3}, x_{7}, \cdots, x_{4 s-5}, x_{4 s+3}, \cdots, x_{4 n-1}\right),
$$

$\left(d^{0} a=1, d^{0} x_{i}=i, 1 \leqq i \leqq 4 n\right)$. There are analogous results for $P S O(2 r)$ 
and for the quotients of $S U(n)$, which we do not recall here (see [5, $\S 10])$.

$H^{*}\left(S O(n), Z_{2}\right)$ has a simple system of universally transgressive elements $\left(h_{i}\right)\left(d^{0} h_{i}=i, i=1, \cdots, n-1\right)$, with the relations

$$
S q^{i} h_{j}=\left(\begin{array}{c}
j \\
i
\end{array}\right) h_{i+j} \quad(i \leqq j, i+j \leqq n-1), S q^{i} h_{j}=0 \text { otherwise. }
$$

In particular, $h_{i} \cdot h_{i}=h_{2 i}$ if $2 i \leqq n-1$, and $h_{i} \cdot h_{i}=0$ otherwise. $H^{*}\left(P S O(2 n), Z_{2}\right)$ is derived from this in the same way as $H^{*}(P S p(n)$, $Z_{2}$ ) from $H^{*}\left(S p(n), Z_{2}\right)$.

Finally, let $s(n)$ be the integer such that $2^{s(n)-1}<n \leqq 2^{s(n)}$. Then $H^{*}\left(\right.$ Spin $(n), Z_{2}$ ) has a simple system of generators whose degrees form a sequence obtained from $3,4, \cdots, n-1$ by erasing all powers of 2 and adding $2^{s(n)}-1$ (one generator for each degree). The $S q^{i}$ are also known for the greater part $[5, \S 13]$.

(11.5) The groups of the structure $E_{6}\left(r e s p . E_{7}, E_{8}\right)$ have no p-torsion for $p \geqq 7$ (resp. $p \geqq 11$ ) (see [7]) and the group of structure $E_{8}$ has 2torsion $[11$, see $\$ 12]$.

(11.6) Let $G$ be compact, connected, simply connected, simple and non-abelian. Then $H_{3}(G, Z)=Z$.

This is deduced by Hurewicz's isomorphism theorem from $\pi_{1}(G)$ $=\pi_{2}(G)=0, \pi_{3}(G)=Z$ (see $\left.\S 18\right)$.

(11.7) We have already pointed out that the homology ring (the multiplication being the Pontrjagin product) $\bmod p$ is an exterior algebra when there is no $p$-torsion, in particular for $p=0$. It follows also from $\S 7$ and (11.4) that $H_{*}\left(S O(n), Z_{2}\right), H_{*}\left(G_{2}, Z_{2}\right), H_{*}\left(F_{4}, Z_{2}\right)$ are exterior algebras generated by elements of degrees $(1,2, \cdots$, $n-1),(3,5,6),(3,5,6,15,23)$ respectively, (see [5], and also [56] for $S O(n))$.

Finally, we point out that many results on the classical linear groups are particular cases of theorems on Stiefel manifolds (see the references given above).

12. Remarks on cohomology of Lie groups and on Weyl groups. Many of the results on the torsion of Lie groups listed in $\$ 11$ are established by use of special properties of the individual groups. However even if the three last exceptional groups could be handled in the same way, this would not constitute a fully satisfactory solution of the problem, the ultimate goal being to arrive at a systematic procedure for computing cohomology from the group theoretical, or infinitesimal, properties. For real cohomology this is attained by Chevalley's theorem (see §9) which relates Betti numbers and in- 
variants of the Weyl group. Another method was already contained in E. Cartan's work [16], but it led to computations too cumbersome to be applied to the last 4 exceptional groups. No such result is yet available in cohomology $\bmod p$ for $p \neq 0$, though there is evidence of relations between torsion and group-theoretical properties. An example [7] is the theorem stating that if the prime $p$ does not divide the order of the Weyl group of a compact connected Lie group $G$, then $G$ has no $p$-torsion. For $E_{6}, E_{7}, E_{8}$ the orders of the Weyl groups are $2^{7} \cdot 3^{4} \cdot 5,9 ! \cdot 8,10 ! \cdot 3 \cdot 2^{6}$ respectively, whence the first part of (11.5). Also all results known so far agree with the following conjecture: If $p$ is greater than the coefficients of the highest root, expressed as linear combination of fundamental roots, then the simply connected group $G$ has no $p$-torsion. For the simply connected representatives of the structures $E_{6}, E_{7}, E_{8}$ this would imply no $p$-torsion for $p \geqq 5, p \geqq 5, p \geqq 7$ respectively.

In this connection, let us also mention that if a compact connected Lie group of rank $r$ contains an abelian subgroup isomorphic to $\left(Z_{p}\right)^{k}$ with $k>r$, then it has $p$-torsion [11]; in application $E_{8}$ has 2torsion. Also, if $H^{*}\left(G, Z_{2}\right)$ has a simple system of $s$ universally transgressive generators, then it does not contain a subgroup isomorphic to $\left(Z_{2}\right)^{t}$ with $t>s$; the upper bound of $t$ is equal to $s$ for $G=U(n)$, $S U(n), S p(n), S O(n)$ (any $n), G_{2}, F_{4}$. In other cases, the connection between abelian subgroups of type $(p, \cdots, p)$ and $p$-torsion is not as simple. Anyway, in all examples known to the author, it seems to be true that $G$ has $p$-torsion if and only if it has an abelian subgroup of type $(p, \cdots, p)$ not contained in a maximal torus.

The connections between Weyl groups and homology suggest some problems about the former. Viewed as a transformation group of the universal covering $R^{r}$ of a maximal torus $T$ of a compact connected Lie group $G$ of rank $r$, the Weyl group is a finite linear group, generated by reflections in hyperplanes, which is moreover "crystallographic," i.e. it leaves a lattice of rank $r$ invariant or, equivalently, is represented by matrices with integral coefficients in a suitable basis. Conversely, every crystallographic group generated by reflections is a Weyl group, as was checked by E. Stiefel [74] and later on given an a priori proof by C. Chevalley (C. R. Acad. Sci. Paris vol. 227 (1948) pp. 1136-1138) and Harish-Chandra (Trans. Amer. Math. Soc. vol. 70 (1951) pp. 28-96, Theorem 1). We recall that two locally isomorphic, not isomorphic, groups have isomorphic Weyl groups but different lattices [74]. We have seen ( $\$ 9)$ that the ring of invariant polynomials with real coefficients of a Weyl group $W(G)$ has $r$ algebraically independent generators $(r=$ dimension of the vector space 
on which $W(G)$ operates, i.e., the rank of $G)$. This can in fact be proved directly for all finite groups generated by reflections (Chevalley), crystallographic or not. In the crystallographic case, it does not seem to be known whether a similar result is valid for invariants with integral coefficients in the symmetric algebra over $Z$ of an invariant lattice: also, the invariants of a finite group generated by reflections of a vector space over a field of characteristic $p$ have not yet been considered except to prove that Chevalley's theorem holds true for a group obtained by "reduction $\bmod p$ " from a Weyl group $W$, given by integral matrices, when $p$ is prime to the order of the group [7].

Added in proof. For Chevalley's theorem, see a forthcoming note of his in the American Journal of Mathematics.

An interesting question about Weyl groups also arises in connection with a result of Bott [13]. Let $G$ be a compact, connected and simply connected Lie group and $\Omega_{G}$ the space of closed paths on $G$, with a fixed origin. Then Bott has proved that $\Omega_{G}$ has no torsion, and moreover he gives an explicit method to get the Betti numbers of $\Omega_{G}$ out of the "diagram" of $G$ (i.e., essentially the planes of reflections of the elements of $W(G)$ and the invariant lattice). On the other hand, these Betti numbers can be read off from the degrees of the invariants of $W(G)$ with real coefficients (using Chevalley's theorem and spectral sequences arguments). Thus a connection between these degrees and the diagram which so far has been proved only with the help of rather difficult topological methods. For more details and other problems about these finite groups, we refer to G. C. Shephard [72].

13. Cohomology of homogeneous spaces. The most comprehensive result on the real cohomology of a homogeneous space is due to $H$. Cartan [20] (see $[2, \S 26]$ for a topological proof). It says that $H^{*}(G / U, R)$ ( $G$ compact connected, $U$ closed connected) is isomorphic to the cohomology ring of the tensor product $H^{*}\left(B_{U}, R\right)$ $\otimes H^{*}(G, R)$ relative to a certain coboundary operator completely (and explicitly) determined by the transgression in $E_{G}$ and by a homomorphism which turns out to be equivalent to $\rho^{*}(U, G)$. Many particular results obtained previously by Samelson, Leray, Koszul can be derived from it, either directly or using Koszul's theory of homology of $S$-modules [42]. Particular cases of H. Cartan's theorem are to be found in [65].

Cartan's theorem shows how to compute $H^{*}(G / U, R)$, knowing $H^{*}(G, R), H^{*}(U, R)$, and $\rho^{*}(U, G)$, i.e. in the last analysis out of group theoretical information, namely the invariants of the Weyl groups of $G$ and $U$ and the position of a maximal torus of $U$ in a 
maximal torus of $G$. This reduction of cohomology theory to group theory has not been yet achieved in characteristic $p \neq 0$ (except however for characteristic classes of the tangential structure, see $\$ 14)$; the best tools presently available are spectral sequences; one of them [2, Théorème 22.1] is so to say a weak analog of $\mathrm{H}$. Cartan's algebra mentioned above and it seems possible that $\mathrm{H}$. Cartan's theorem is also valid $\bmod p$ when $G$ and $U$ have no $p$-torsion or, more generally, when their cohomology rings mod $p$ are generated by universally transgressive elements.

The proof of (C) in $\$ 9$ also gives information on $H^{*}\left(G / U, Z_{p}\right)$ when $G$ and $U$ have the same rank and no $p$-torsion $[2, \S \S 29,30]$. In that case $G / U$ has no $p$-torsion, $H^{*}\left(G / U, Z_{p}\right)$ is isomorphic to the quotient of $I_{U} \otimes Z_{p}$ by the ideal generated by the elements of strictly positive degrees in $I_{G} \otimes Z_{p}$, and its Poincaré polynomial is

$$
P_{p}(G / U, t)=\left(1-t^{2 m_{1}}\right) \cdots\left(1-t^{2 m_{r}}\right) \cdot\left(1-t^{2 n_{1}}\right)^{-1} \cdots\left(1-t^{2 n_{r}}\right)^{-1},
$$

$\left(2 m_{1}-1, \cdots, 2 m_{r}-1\right)$ (resp. $\left.2 n_{1}-1, \cdots, 2 n_{r}-1\right)$ being the degrees of the primitive elements in $H^{*}\left(G, Z_{0}\right)$ (resp. $\left.H^{*}\left(U, Z_{0}\right)\right)$. This is valid in particular for $p=0$ and the last equality is then the Hirsch formula, conjectured by G. Hirsch, proved by H. Cartan-J. L. Koszul $[20 ; 42]$ and by J. Leray [49] (see also $[2, \S 26]$ ). Also, if only $U$ has no $p$-torsion then $G / U$ has no $p$-torsion and (13.1) is again valid although the multiplicative structure may be rather different. Similar statements are valid for integral cohomology when the assumption "no p-torsion" is replaced by "no torsion." These results allow us to describe rather completely the cohomology of certain classical coset spaces, like complex Grassmann manifolds or complex quadrics [2, $\S 31]$.

In $\S 9$ it was pointed out that results similar to (C) hold for the cohomology mod 2 of the orthogonal groups and their classifying spaces if maximal tori are replaced by maximal abelian subgroups of type $(2, \cdots, 2)$. This analogy can be pushed further and leads in certain cases to results paralleling those above for $H^{*}\left(G / U, Z_{2}\right)$ when $G$ and $U$ have the same "2-rank," i.e., have a maximal abelian subgroup of type $(2, \cdots, 2)$ in common. One gets a "Hirsch formula" mod 2 , in which degrees of primitive elements are replaced by degrees of the members of simple systems of universally transgressive generators [3].

At the other extreme so to say is the case where $U$ is a circle. Koszul had shown that $P_{0}\left(G / T^{1}, t\right)$ (G compact semi-simple) is obtained from $P_{0}(G, t)$ simply by writing $\left(1+t^{2}\right)$ for $\left(1+t^{3}\right)$; this follows also from H. Cartan's theorem, which moreover gives the multiplica- 
tive structure of $H^{*}\left(G / T^{1}, R\right)$. The cohomology mod $p$ of that quotient can also be investigated when $H^{*}\left(G, Z_{p}\right)$ has a simple system of universally transgressive generators; here $P_{p}(G / U, t)$ is $P_{p}(G, t)$ with one of the factors $\left(1+t^{2 k+1}\right)$ replaced by $\left(1+t+\cdots+t^{2 k}\right)$, but $k$ may now be $\neq 1$ and its value depends in fact on the position of subgroup in the group. This case has not yet been treated in the literature but it is quite analogous to, only slightly simpler than, the study of the quotient $G / Z_{p}$, which is done in $[5, \S 10]$; the common feature of these two problems is that $H^{*}\left(B_{U}, Z_{p}\right)$ is or is "almost" a ring of polynomials with one generator.

14. Characteristic classes of homogeneous spaces. A coset space $G / U$ is a differentiable manifold; hence we may consider the Pontrjagin or Stiefel-Whitney classes of its tangential structure, or also its Chern classes when it carries an almost complex or a complex analytic structure invariant under $G$. It turns out that they can be expressed by means of group theoretical invariants, namely, by roots [9]. We content ourselves with some brief indications, assuming familiarity of the reader with the theory of roots of complex or compact semisimple Lie algebras (see e.g. $[26 ; 74 ; 81])$. The roots are usually defined as linear forms on the Lie algebra of a maximal torus $T$ but they can in a natural way be identified with elements of $H^{1}(T, Z)$, and, via transgression, with elements of $H^{2}(G / T, Z)$. Now to any system of positive roots $\left(a_{i}\right),(i=1, \cdots, m, \operatorname{dim} G=\operatorname{dim} T+2 m)$, there is associated a complex analytic structure on $G / T$ (about which more will be said in $\$ 15$ ), invariant under $G$. Its $i$ th Chern class is then the $i$ th elementary symmetric function in the $a_{i}$ 's. If $G$ and its closed connected subgroup $U$ have the same rank and if $G / U$ carries an invariant complex analytic structure, then the map $f^{*}$ induced by the natural projection of $G / T$ onto $G / U$ maps the $i$ th Chern class on the $i$ th elementary symmetric function in the roots of $G$ which are not roots of $U$. Similarily, the Pontrjagin classes are symmetric functions in squares of roots (and vanish for $G / T$ ). When $G$ and $U$ have different ranks, one must take the roots of $G$ with respect to a maximal torus of $U$. The Stiefel-Whitney classes $(\bmod 2)$ may be also connected with symmetric functions not exactly in the roots but in what may be called the "2-roots," i.e., essentially the characters of a maximal abelian subgroup of type $(2,2, \cdots, 2)$ in the adjoint representation. These facts are interesting for $G / T$, where they allow us to establish relations between topological properties, theorem of Riemann-Roch, and formulas of representation theory [9].

15. $G / T$ and related spaces. Cellular decompositions. We say that 
a compact manifold has a cellular decomposition if there is a partition of $M$ into a finite number of embedded submanifolds $M_{i}$, the "open cells," each homeomorphic to some affine space, and having a settheoretical boundary made up of open cells of strictly smaller dimensions. The closures of the open cells then form a cellular decomposition of $M$ in the sense of combinatorial topology. In the same way a compact complex analytic manifold has a complex analytic cellular decomposition if the $M_{i}$ are embedded complex analytic submanifolds, bi-holomorphically homeomorphic to complex affine spaces. In his Thesis [33] Ehresmann constructed complex analytic cellular decompositions for certain algebraic homogeneous spaces (Grassmann manifolds, complex quadrics, $S O(2 n) / U(n), S p(n) / U(n)$, and some others), using Schubert systems. This can be done in fact for all algebraic varieties which admit a transitive compact semi-simple group of complex analytic homeomorphisms, by a group theoretical method [6]. The manifolds in question are the quotients $G / U$, where $G$ is compact semi-simple and $U$ is the centralizer of a toral subgroup of $G$. The complexification $G^{c}$ of $G$ also operates on $G / U$, and the latter may be identified with a quotient of $G^{c}$ by a complex subgroup $V$ containing a maximal connected solvable subgroup $L$ of $G^{c}$ (and, conversely, every quotient $G^{c} / V$ with $V \supset L$ is algebraic); the group $L$ is generated by a Cartan subalgebra and the root vectors corresponding to a system of positive roots. The open cells are then just the orbits of $L$ acting in the obvious way on $G^{c} / V$; they are also birationally and biregularly equivalent to complex affine spaces, and it follows that these coset spaces are rational varieties; this last fact was first proved by M. Goto [37] and, as a matter of fact, precisely by construction of the open cell of highest dimension. Among these spaces we find $G / T$, where $T$ is as usual a maximal torus of $G$, which may also be written $G^{c} / L$. In this case the cells are in 1-1 correspondence with the elements of the Weyl group $W(G)$ of $G$, which implies that the Euler-Poincaré characteristic $\chi(G / T)$ of $G / T$ is equal to the order of the Weyl group. This gives a new proof of a result of A. Weil (C. R. Acad. Sci. Paris vol. 200 (1935) pp. 518-520) which was later proved again by Hopf-Samelson [39]: more precisely [39] gives $\chi(G / U)$ for any coset space $G / U$ with $G$ compact, but this follows by easy fiber bundle arguments once $\chi(G / T)$ is known. (Added in proof. The previous cellular decomposition of $G / T$ is also obtained in a paper by C. Chevalley on simple groups, to appear in The Tohoku Mathematical Journal.)

Since they carry a complex analytic cellular decomposition, the coset spaces considered above have no torsion, a fact first proved by 
R. Bott [13], who moreover gives an expression of the Poincare polynomial of the space computable out of the diagram of singular elements of $G$. Both procedures are group theoretical but have the disadvantage of not yielding any information on the cup-product in $H^{*}(G / U, Z)$. For $G / T$ another approach has been devised by Bott and Samelson [14]. They first construct a space, say $Y_{G}$, of the same dimension as $G / T$, which is a multiple fibering with 2-dimensional spheres as fibers, and whose cohomology ring is determined by the Cartan integers of $G$. Then they prove the existence of a map $f$ of degree 1 of $Y_{G}$ onto $G / T$, and show that the 1-1 image of $f^{*}$ is a direct summand completely characterized by the expressions of the roots of $G$ as linear combinations of fundamental roots. It may be hoped that this will for instance allow one to prove $a$ priori a fact checked by the author [7] for all simple groups $G$ : If $p$ is strictly greater than the coefficients of the dominant root, expressed as sum of fundamental roots, then $H^{*}\left(G / T, Z_{p}\right)$ is generated by 1 and by $H^{2}\left(G / T, Z_{p}\right)$, and, consequently, $B_{G}$ has no $p$-torsion, when $G$ is simply connected.

Ehresmann [34] and Nordon [61] have studied cellular decompositions of some real algebraic manifolds, notably the real "flag" manifolds $O(n) / O\left(n_{1}\right) \times \cdots \times O\left(n_{k}\right) \quad\left(n_{1}+\cdots+n_{k}=n\right)$. A new difficulty arises because the cells may have all possible dimensions and are not always cycles, so that incidence numbers must be computed. It was shown that these cells are cycles mod 2 and that all torsion coefficients of the integral homology groups are equal to 2 . These cells may also be defined as orbits, in essentially the same way as in the complex case, and it might have some interest to know whether this method extends to a wider class of real algebraic coset spaces and if so, whether these spaces have the same torsion properties as the flag manifolds.

16. Homogeneous complex spaces. In connection with the manifolds discussed in $\$ 15$, we now digress slightly to report on recent investigations about homogeneous complex spaces.

A coset space $G / U$ is homogeneous complex (resp. homogeneous kählerian) if it carries a complex analytic structure (resp. and a kählerian metric) invariant under $G$. The quotients $G / U$ ( $G$ compact, $U$ centralizer of a torus) considered in $\$ 15$ are exactly the compact homogeneous kählerian spaces with finite fundamental group and are in fact all simply connected. We have pointed out that they are rational algebraic varieties; they have been studied from a different point of view by $\mathrm{J}$. Tits $[77 ; 78]$.

More generally, Wang [79] has determined all compact simply con- 
nected homogeneous complex spaces. These are precisely the even dimensional quotients $G$ / $U$ where $G$ is compact and $U$ is (locally) the product of a torus $T^{s}$ by the semi-simple part of the centralizer of some torus (containing $T^{s}$ of course, but possibly bigger). Among them we find the even dimensional compact groups, a particular case also discussed by $H$. Samelson [68]. In the nonalgebraic case, not much is known about compact homogeneous complex spaces with infinite fundamental group: if it is algebraic, then it is globally a direct product of a rational coset space (see above) by an algebraic complex torus (not yet published by the author); if it is homogeneous kählerian, then it is locally the product of a torus by a simply connected kählerian homogeneous space [52]; if the compact complex manifold is "complex parallisable", it is the quotient of a complex Lie group by a discrete subgroup [80].

In the noncompact case, some results have been obtained about homogeneous kählerian or homogeneous symplectic (i.e. carrying an invariant nondegenerate closed exterior 2-form) coset spaces. For instance [6], if $G$ is semi-simple, with maximal compact subgroup $K$, then $G / U$ is homogeneous kählerian if and only if $U$ is compact, centralizer of a torus, and $G / K$ is hermitian symmetric. (For hermitian symmetric spaces, see [10].) Related results are obtained by Koszul [43] who investigates necessary conditions for a homogeneous space to carry a nondegenerate 2 -form derived from an invariant volume element, in the same way as Bergmann's metric is derived from the kernel function. Under that assumption, he moreover proves e.g. that if $G$ is effective, then its center must be discrete.

A bounded domain in $C^{n}$ carries a kählerian metric (the Bergmann metric) invariant under all complex analytic homeomorphisms; hence it is homogeneous kählerian whenever it is homogeneous complex. E. Cartan determined all those which are symmetric ([17], see also [10]). They are products of irreducible ones, the latter ones being exactly the quotients $G / K$ ( $G$ simple noncompact, $K$ maximal compact subgroup) where $K$ has a nondiscrete center. To be more accurate, E. Cartan had checked case by case (except for possibly two cases) that all these spaces are equivalent to bounded domains; recently this has been proved $a$ priori, by a general argument, for all of them by Harish-Chandra (yet unpublished). E. Cartan posed the question as to whether there are bounded homogeneous domains which are not symmetric. Only partial answers have been given so far: the bounded domain $G / U$ is symmetric if the linear isotropy group is irreducible [50] (also contained in [43]) or if $G$ is semi-simple [6;43]. 
17. Noncompact Lie groups and their coset spaces. A fundamental theorem due to E. Cartan-Malcev-Iwasawa (see [67] for references) states that a connected Lie group $G$ is homeomorphic to the direct product of a maximal compact subgroup $K$ by a euclidian space. More precisely, there exists a subspace $F$ homeomorphic to $R^{m}$ such that each $g \in G$ may be written in one and only one way as product $k \cdot f$, with $k \in K, f \in F$, depending continuously on $g$. We write $G=K \cdot F$ or $G=K \cdot R^{m}$. Moreover, every compact subgroup of $G$ is conjugate by an inner automorphism to a subgroup of $K$.

The circumstances are more complicated for a coset space $G / U$, even when $U$ is connected. Let us write

$$
G=K \cdot R^{s}, \quad U=L \cdot R^{t}
$$

( $K$, resp. $L$, compact maximal in $G$, resp. $U, K \supset L)$.

Then $s \geqq t$ and $G / U$ has the same homological properties as $K / L \times R^{s-t}$ [4], and in fact $K / L$ is a deformation retract of $G / H$ [58]: this implies in particular that $G / U$ has at most 2 ends in Freudenthal's sense [4]. The space $G / U$ is homeomorphic to the product $K / L \times R^{s-t}$ when $s-t=1$ [4] or also when $G$ is solvable (Chevalley, see [57]; $G / U$ is then a product of circles and straight lines). It is natural to ask whether the above decompositions of $G$ and $U$ may be chosen in a "coherent" way, i.e., with $R^{t} \subset R^{s}$. This apparently difficult question has not yet been fully answered. Mostow [58] has shown, under a rather often fulfilled condition, which might conceivably be superfluous, that there exist subspaces $E, F$ of $G$, homeomorphic to euclidian spaces, generated, by means of the exponential, by subspaces of the Lie algebra of $G$ invariant under the inner automorphisms of $L$, such that

$$
G=K \cdot F \cdot E, \quad U=L \cdot F .
$$

It follows that $G / U$ has a fibering with base $K / L$ and typical fiber $E=R^{s-t}$. Mostow's condition is that $U$ be "self-adjoint modulo the radical $R(G)$ of $G$," i.e., $U R(G) / R(G)$ must be invariant under one characteristic involutive automorphism of the semi-simple group $G / R(G)$. This condition is always fulfilled when $U$ is semi-simple [59]. It has also been shown in [58] that if $G / U$ is acyclic (i.e., as is easily seen, if and only if $K=L$ ) then $G / U$ is homeomorphic to a euclidian space (without any other condition on $U$ ). Some acyclic homogeneous spaces are discussed in [15].

The quotients $G / U$, with $G$ connected noncompact, $U$ nonconnected, have been investigated so far only when $G$ is nilpotent [54; $55 ; 60]$, or solvable [57]. In the former case $G / U$ is the product of 
a euclidian space by a compact homogeneous space of a nilpotent group; in the latter case there exists a subgroup $U^{*}$ of finite index of $U$, containing the commutator group of $U$, such that $G / U^{*}$ is the product of a euclidian space by a compact coset space of a solvable group. The example of the Möbius strip shows that one may have to go to a covering to get a product decomposition. Since a connected and simply connected solvable group $G$ is homeomorphic to euclidian space (see e.g. [18]), it is immediately seen that $G / U$ is a classifying space, in the sense of $\S 8$, for its fundamental group $U / U_{0}$, ( $U_{0}$ connected component of the identity in $U$ ). It follows then from standard facts of fiber bundle theory that if $G / U$ and $G^{\prime} / U^{\prime}\left(G, G^{\prime}\right.$ simply connected solvable) have isomorphic fundamental groups, then they have the same homotopy type. If they are moreover compact, then they are actually homeomorphic [57]. When $G, G^{\prime}$ are nilpotent simply connected, and $U, U^{\prime}$ are discrete, this homeomorphism is induced by an isomorphism of $G$ onto $G^{\prime}$ carrying $U$ onto $U^{\prime}$ [54], but this need not be so in the solvable case. Malcev has proved that a nilpotent Lie group $G$ has a discrete subgroup $U$ with compact quotient $G / U$ if and only if its Lie algebra has rational constants of structure, relative to a suitable basis; this is not generally true for solvable groups, in fact, the affine group of the real line has no discrete subgroup with a compact quotient, as may be seen without difficulty.

A compact coset space $G / U$ with infinite fundamental group is not in general the quotient of a compact Lie group, and little is known about its homological properties, except for nilpotent $G$. In that case $G / U$ is orientable and its Euler-Poincare characteristic is $\geqq 0$ [60]; the second property is also shared by the quotients of compact groups [39] and it is not known whether this is true for all compact homogeneous spaces. For nilpotent $G$ again, $H^{*}(G / U, R)$ is isomorphic to the cohomology ring of the Lie algebra of $G$ modulo the Lie algebra of $U$, in the sense of Chevalley-Eilenberg; this had been proved by Matsushima [55] in dimensions 1, 2 and was generalized by K. Nomizu [60] for all dimensions. Here again, easy examples show that it may be wrong for solvable $G$. Even in the nilpotent case, $H^{*}(G / U, R)$ is not always isomorphic to the cohomology ring of invariant differentiable forms [60], in contrast with E. Cartan's result [16] about compact groups $G$.

A discrete subgroup is the fundamental group of the quotient of a connected nilpotent (resp. solvable) Lie group if and only if it is a torsion free finitely generated nilpotent group [54], (resp. the extension of a torsion free finitely generated nilpotent group by a 
finitely generated free abelian group (H. C. Wang, Discrete subgroups of solvable groups I, to appear in the Annals of Mathematics)).

18. Homotopy groups of Lie groups. The $n$th homotopy group $\pi_{n}(X)$ or $\pi_{n}(X, P)$ of a space $X$ is the set of all homotopy classes of continuous maps of the $n$-dimensional sphere $S_{n}$ into $X$, sending a fixed point of $S_{n}$ onto a fixed point $P \in X$, endowed with a suitable law of composition (see e.g. [73]), which is commutative for $n \geqq 2$. The group $\pi_{1}(X)$ is the fundamental group of $X$, it is also abelian when $X$ is a topological group (or an $H$-space). $\pi_{n}(X \times Y)$ is the direct sum of $\pi_{n}(X)$ and $\pi_{n}(Y)$; also a space and a covering space have isomorphic $n$th homotopy group for $n \geqq 2$. Hence, taking into account the theorem recalled at the beginning of $\$ 17$, it is enough, when dealing with Lie groups, to consider compact semi-simple (or even simple) Lie groups.

In the introduction, we mentioned $\mathrm{H}$. Weyl's theorem to the effect that $\pi_{1}(G)$ is finite for $G$ compact connected, semi-simple ([81]; see also [69] and [67] for references to other proofs). Extending Weyl's argument further, E. Cartan [18] showed that $\pi_{2}(G)=0$. It follows also directly from the fact that the space of loops on $G$ has no torsion (for simply connected $G$ ) [13]; it can also be obtained by an easy homotopy sequence argument from the absence of torsion on $G / T$, discussed in $\$ 15$. Bott's result also implies that $\pi_{3}(G)=Z$, for $G$ compact connected, simple, non-abelian.

By use of Morse theory, Bott and Samelson have obtained the following result on $\pi_{4}(G)$, for $G$ compact connected, simply connected, simple. Let $T$ be a maximal torus, $R^{r}$ its universal covering, $\Gamma$ the inverse image of the identity of $T$ in $R^{r}$, and let $a$ be the dominant root with respect to some lexicographic order of the roots of $G$. Then $\pi_{4}(G)=Z_{2}$ (resp. $\pi_{4}(G)=0$ ) if the hyperplane $a=1$ has a point (resp. no point) of $\Gamma$ (yet unpublished).

It was pointed out several times that the cohomology ring over the reals of a compact connected Lie group $G$ is an exterior algebra generated by $r$ elements $(r=\operatorname{rank} G)$ of odd degrees, say $\left(m_{i}\right)(1 \leqq i \leqq r)$. In other words, $H^{*}(G, R)$ is isomorphic to the real cohomology ring of a product of spheres of dimensions $m_{1}, \cdots, m_{r}$, which will be denoted by $X_{G}$. This of course leads to the problem of knowing to what extent $G$ and $X_{G}$ have similar properties. L. S. Pontrjagin [63] showed that $S U(n)(n \geqq 3)$, which has the same integral cohomology ring as $X_{S U(n)}$, is not the product of $S_{3}$ by some space, hence is not homeomorphic to $X_{S U(n)}$. His proof used homotopy, but the theorem can also be obtained in the framework of homology, by showing that there is a nonzero Steenrod square $S q^{2}$ in $H^{*}\left(S U(n), Z_{2}\right)$. In a similar 
way, Steenrod's reduced powers allow one to show that $S p(n)$ $(n \geqq 2)$ is not homeomorphic to $X_{S_{p(n)}}[12]$. The groups $G_{2}, F_{4}, E_{8}$, having torsion, are not homeomorphic to the corresponding products of spheres, and it is rather likely to be also true for $E_{6}, E_{7}$ so that finally it seems highly probable that no simple group of rank $>1$ is homeomorphic to a product of spheres.

However, $G$ and $X_{G}$ have many common properties, discovered by J-P. Serre [70]. For instance, there exists a map of $G$ into $X_{G}$ which induces an isomorphism of $\pi_{n}(G) \otimes Z_{0}$ onto $\pi_{n}\left(X_{G}\right) \otimes Z_{0}(G$ is compact semi-simple, connected). Known results on spheres show then that the rank of $\pi_{n}(G)$ is equal to the number of $m_{i}$ (as defined above) equal to $n(n \geqq 1)$; in particular, $\pi_{n}(G)$ is finite if $n \neq m_{i}(1 \leqq i \leqq r)$, e.g., if it is even. Let $G$ be compact connected, simply connected semi-simple, of dimension $m$ and rank $r$. Then if $p \geqq m / r-1$, there is a map of $X_{G}$ into $G$ which induces an isomorphism of the $p$-primary component of $\pi_{n}\left(X_{G}\right)$ onto the $p$-primary component of $\pi_{n}(G)(n \geqq 2)$ ( $[70, \S 5]$, we have also included the exceptional groups in the statement by making use of [7]); moreover, if $G$ is a classical group, such a map exists only if $p \geqq m / r-1$ [70]; however, the latter inequality is not necessary for the exceptional groups, because it follows from Toda's result $\pi_{10}\left(G_{2}\right)=Z_{3}$ (see below) that there is such a map for $G=G_{2}$, and $p=3$ (see [70, last remark]).

The foregoing gives all known homotopy properties having a general character. Other results have been obtained for the classical groups, $G_{2}, F_{4}$ by extensive use of homotopy theory. We summarize the main ones.

First, we remind the reader of the isomorphisms (5.1) and we recall the isomorphisms

$$
\begin{aligned}
\pi_{i}(S O(m)) & =\pi_{i}(S O(n)) & (n, m \geqq i+2) \\
\pi_{i}(S U(m)) & =\pi_{i}(S U(n)) & (n, m \geqq(i+1) / 2), \\
\pi_{i}(S p(m)) & =\pi_{i}(S p(n)) & n, m \geqq(i-1) / 4), \\
\pi_{i}\left(G_{2}\right) & =\pi_{i}(\operatorname{Spin}(7)) & (i \leqq 5), \\
\pi_{i}\left(F_{4}\right) & =\pi_{i}(\operatorname{Spin}(9)) & (i \leqq 6), \\
\pi_{i}(\operatorname{Spin}(9)) & =\pi_{i}(\operatorname{Spin}(7)) & (i \leqq 13),
\end{aligned}
$$

which follow from the homotopy sequence of the fiberings:

$$
\begin{aligned}
S O(n) / S O(n-1) & =S_{n-1}, & S U(n) / S U(n-1) & =S_{2 n-1}, \\
S p(n) / S p(n-1) & =S_{4 n-1}, & \operatorname{Spin}(7) / G_{2} & =S_{7}, \\
F_{4} / \operatorname{Spin}(9) & =W, & \operatorname{Spin}(9) / \operatorname{Spin}(7) & =S_{15}
\end{aligned}
$$


where $W$ is the projective plane over the Cayley numbers (see [1] for the last three fiberings).

The following table lists the groups $\pi_{i}(G)$ for $i \leqq 13, G$ a classical group of $G_{2}, F_{4}$ as given to the author by $\mathrm{H}$. Toda. We write $m$, for $Z_{m}$ and $Z$ respectively. Some of these results had been established previously by several authors: For $S U(2)=S_{3}$, see notably $[71 ; 73]$; H. Toda, Jour. Inst. Polyt. Osaka City Univ. vol. 3 (1952) pp. 4382, C. R. Acad. Sci. Paris vol. 240 (1955) pp. 42-44, 147-149; J-P. Serre, C. R. Acad. Sci. Paris vol. 234 (1952) pp. 1243-1245. For $\pi_{4}, \pi_{5}, \pi_{6}$ of the classical groups, see $[73 ; 32 ; 12]$ respectively. The groups $\pi_{7}(S O(n)), \pi_{8}(S O(n))$ have been determined by Serre [71], Sugawara $[75 ; 76]$, Paechter (not yet published), the two last named authors giving also the value of $\pi_{9}(S O(n))$. In [76] we also find partial information on $\pi_{10}(S O(n)), \pi_{11}(S O(n))$ and [12] gives also some results on $p$-primary components of higher homotopy groups of the classical groups.

\begin{tabular}{lc|cccccccccc} 
& & 4 & 5 & 6 & 7 & 8 & 9 & 10 & 11 & 12 & 13 \\
\hline$S U$ & $(2)$ & 2 & 2 & 12 & 2 & 2 & 3 & 15 & 2 & $2+2$ & $2+4+2$ \\
$S U$ & $(3)$ & 0 & $\infty$ & 6 & 0 & 12 & 3 & 30 & 4 & 60 & 6 \\
$S U$ & $(4)$ & 0 & $\infty$ & 0 & $\infty$ & 24 & 2 & $120+2$ & 4 & 60 & 4 \\
$S U$ & $(5)$ & 0 & $\infty$ & 0 & $\infty$ & 0 & $\infty$ & 120 & 0 & 360 & 4 \\
$S U$ & $(6)$ & 0 & $\infty$ & 0 & $\infty$ & 0 & $\infty$ & 3 & $\infty$ & 720 & 0 \\
$S U$ & $(7)$ & 0 & $\infty$ & 0 & $\infty$ & 0 & $\infty$ & 3 & $\infty$ & 0 & $\infty$ \\
$S p$ & $(2)$ & 2 & 2 & 0 & $\infty$ & 0 & 0 & 120 & 2 & $2+2$ & $4+2$ \\
$S p$ & $(3)$ & 2 & 2 & 0 & $\infty$ & 0 & 0 & 3 & $\infty$ & 2 & 2 \\
$S O$ & $(8)$ & 0 & 0 & 0 & $\infty+\infty$ & $2+2+2$ & $2+2+2$ & $24+24$ & $\infty+2$ & 0 & $2+2$ \\
$S O$ & $(9)$ & 0 & 0 & 0 & $\infty$ & $2+2$ & $2+2$ & 24 & $\infty+2$ & 0 & 2 \\
$S O(10)$ & 0 & 0 & 0 & $\infty$ & 2 & $\infty+2$ & 12 & $\infty$ & 12 & 2 \\
$S O(11)$ & 0 & 0 & 0 & $\infty$ & 2 & 2 & 6 & $\infty$ & 2 & $2+2$ \\
$S O(12)$ & 0 & 0 & 0 & $\infty$ & 2 & 2 & 3 & $\infty+\infty$ & $2+2$ & $2+2$ \\
$S O(13)$ & 0 & 0 & 0 & $\infty$ & 2 & 2 & 3 & $\infty$ & 2 & 2 \\
$S O$ & $(14)$ & 0 & 0 & 0 & $\infty$ & 2 & 2 & 3 & $\infty$ & 0 & $\infty$ \\
$S O$ & $(15)$ & 0 & 0 & 0 & $\infty$ & 2 & 2 & 3 & $\infty$ & 0 & 0 \\
$G_{2}$ & 0 & 0 & 3 & 0 & 2 & 6 & 3 & $\infty+2$ & 0 & 3 \\
$F_{4}$ & 0 & 0 & 0 & 0 & 2 & 2 & 0 & $\infty+2$ & 0 & 0
\end{tabular}

In this connection, let us mention a question raised by J-P. Serre: As far as homology or infinitesimal theory are concerned, the groups $S O(2 n+1)$ and $S p(n)$ "differ only with respect to the prime 2. " Is it then true that $\pi_{i}(S O(2 n+1))$ and $\pi_{i}(S p(n))$ have isomorphic $p$ primary components for all odd primes? Using the above results, Serre has proved that it is indeed the case for $n=3$. For $n=1,2$ it is of course implied by (5.1). 
Very little is known about homotopy groups of homogeneous spaces, and up to now, only those of the Stiefel manifolds have been subject to rather systematic investigations, see J. H. C. Whitehead, Proc. London Math. Soc. vol. 48 (1944) pp. 243-291, vol. 49 (1947) pp. 479-481; [32]; [44] and a forthcoming paper of G. F. Paechter.

\section{BIBLIOGRAPHY}

As regards the papers published up to 1951 , we refer to [67] for a complete list and mention here mainly those which are connected with the text.

1. A. Borel, Le plan projectif des octaves et les sphères comme espaces homogènes, C. R. Acad. Sci. Paris vol. 230 (1950) pp. 1378-1380.

2. - Sur la cohomologie des espaces fibrés principaux et des espaces homogènes de groupes de Lie compacts, Ann. of Math. vol. 57 (1953) pp. 115-207.

3. - La cohomologie mod 2 de certains espaces homogènes, Comment. Math. Helv. vol. 27 (1953) pp. 165-197.

4. - Les bouts des espaces homogènes de groupes de Lie, Ann. of Math. vol. 58 (1953) pp. 443-457.

5. - Sur l'homologie et la cohomologie des groupes de Lie compacts connexes, Amer. J. Math. vol. 76 (1954) pp. 273-342.

6. - Kählerian coset spaces of semi-simple Lie groups, Proc. Nat. Acad. Sci. U.S.A. vol. 40 (1954) pp. 1147-1151.

7. - Sur la torsion des groupes de Lie, J. Math. Pures Appl., to appear.

8. A. Borel and C. Chevalley, The Betti numbers of the exceptional groups, Memoirs of the American Mathematical Society, no. 14, 1955, pp. 1-9.

9. A. Borel and F. Hirzebruch, On characteristic classes of homogeneous spaces, to appear.

10. A. Borel and A. Lichnerowicz, Espaces riemanniens et hermitiens symétriques, C. R. Acad. Sci. Paris vol. 234 (1952) pp. 2332-2334.

11. A. Borel and J-P. Serre, Sur certains sous-groupes des groupes de Lie compacts, Comment. Math. Helv. vol. 27 (1953) pp. 128-139.

12. - Groupes de Lie et puissances réduites de Steenrod, Amer. J. Math. vol. 73 (1953) pp. 409-448.

13. R. Bott, On torsion in Lie groups, Proc. Nat. Acad. Sci. U.S.A. vol. 40 (1954) pp. 586-588.

14. R. Bott and H. Samelson, On the cohomology ring of $G / T$, Ibid. vol. 41 (1955).

15. L. Calabi, I gruppi semisemplici di Lie che operano sullo spazio euclideo ad $n$ dimensioni, Rend. Mat. e Applicazioni Ser. V vol. 11 (1952) pp. 1-5.

16. E. Cartan, Sur les invariants intégraux de certains espaces homogènes clos et les propriêtés topologiques de ces espaces, Annales de la Société Polonaise de Mathématique vol. 8 (1929) pp. 181-225; Oeuvres completes, Part I, vol. 2, Paris, GauthierVillars, 1952, pp. 1081-1125.

17. - - Sur les domaines bornés homogènes de l'espace de $n$ variables complexes, Abh. Math. Sem. Hamburgischen Univ. vol. 11 (1935) pp. 116-162; Oeuvres complètes, Part. I, vol. 2, Paris, Gauthier-Villars, 1952, pp. 1259-1305.

18. — La topologie des espaces représentatifs des groupes de Lie, Actualités Scientifiques et Industrielles, no. 358, Paris, Hermann, 1936, Oeuvres complètes, Part I, vol. 2, Paris, Gauthier-Villars, 1952, pp. 1307-1330. 
19. H. Cartan, Séminaire de Topologie de l'E.N.S. II, Paris, 1949-1950, (Notes polycopiées).

20. - a. Notions d'algèbre différentielle; application aux groupes de Lie et aux variétés oì opère un groupe de Lie, Colloque de Topologie (espaces fibrés), Bruxelles, 1950, Liège et Paris, 1951, pp. 15-27; b. La transgression dans un groupe de Lie et dans un espace fibré principal, ibid. pp. 57-71.

21. S. S. Chern, Characteristic classes of hermitian manifolds, Ann. of Math. vol. 47 (1946) pp. 85-121.

22. - Topics in differential geometry, Institute for Advanced Study, Princeton, 1951 (mimeographed notes).

23. - On the characteristic classes of complex sphere bundles and algebraic varieties, Amer. J. Math. vol. 75 (1953) pp. 565-597.

24. C. Chevalley, The Betti numbers of the exceptional Lie groups, Proceedings of the International Congress of Mathematicians, Cambridge, Mass., 1950, Providence, American Mathematical Society, 1952, vol. 2, pp. 21-24.

25. C. Chevalley and S. Eilenberg, Cohomology theory of Lie groups and Lie algebras, Trans. Amer. Math. Soc. vol. 63 (1948) pp. 85-124.

26. E. Dynkin, The structure of semi-simple Lie algebras, Uspehi Matematičeskih Nauk (N.S.) vol. 2 (1947) pp. 59-127, translated in Amer. Math. Soc. Translation no. $17,1950$.

27. - Topological invariants of linear representations of the unitary group, C. R. Acad. Sci. URSS (N.S.) vol. 85 (1952) pp. 697-699.

28. - A connection between homologies of a compact Lie group and its subgroups, ibid. vol. 87 (1952) pp. 333-336.

29. - Construction of primitive cycles in compact Lie groups, ibid. vol. 91 (1953) pp. 201-204.

30. - Homological characterisations of homomorphisms of compact Lie groups, ibid. pp. 1007-1009.

31. - Homologies of compact Lie groups, Uspehi Matematiceskih Nauk (N.S.) vol. 5 (1953) pp. 73-120.

31a. - Homological characterizations of homomorphisms of compact Lie groups, Mat. Sbornik N.S. vol. 35 (1954) pp. 129-173.

32. B. Eckmann, Espaces fibrés et homotopie, Colloque de Topologie Algébrique (espaces fibrés), Bruxelles, 1950, Liège et Paris, 1951, pp. 83-89.

33. C. Ehresmann, Sur la topologie de certains espaces homogènes, Ann. of Math. vol. 35 (1934) pp. 396-443.

34. - Sur la topologie de certaines variêtés algébriques réelles, J. Math. Pures Appl. vol. 16 (1937) pp. 69-100.

35. - - Sur la variêté des génératrices planes d'une quadrique réelle et sur la topologie $d u$ groupe orthogonal d $n$ variables, C. R. Acad. Sci. Paris vol. 208 (1939) pp. 321-323.

36. - Sur la topologie des groupes simples clos, ibid. pp. 1263-1265.

37. M. Goto, On algebraic homogeneous spaces, Amer. J. Math. vol. 76 (1954) pp. $811-818$

38. H. Hopf, Ueber die Topologie der Gruppen-Mannigfaltigkeiten und ihrer Verallgemeinerungen, Ann. of Math. vol. 42 (1941) pp. 22-52.

39. H. Hopf and H. Samelson, Ein Satz ibber die Wirkungsräume geschlossener Liescher Gruppen, Comment Math. Helv. vol. 13 (1940-1941) pp. 240-251.

40. I. M. James, A note on factor spaces, J. London Math. Soc. vol. 28 (1953) pp. 278-285. 
41. J. L. Koszul, Homologie et cohomologie des algèbres de Lie, Bull. Soc. Math. France vol. 78 (1950) pp. 65-127.

42. - - Sur un type d'algèbres différentielles en rapport avec la transgression, Colloque de Topologie Algébrique (espaces fibrés), Bruxelles, 1950, Liège et Paris, 1951, pp. 73-81.

43. - Sur la forme hermitienne canonique des espaces homogènes complexes, to appear.

44. T. Kudo, Homological structure of fibre bundles, Jour. Inst. of Polytechnics, Osaka City University vol. 2 (1952) pp. 101-140.

45. J. Leray, Sur la forme des espaces topologiques et sur les points fixes des représentations, J. Math. Pures Appl. vol. 54 (1945) pp. 95-167.

46. - Espaces oì opère un groupe de Lie compact connexe, C. R. Acad. Sci. Paris vol. 228 (1949) pp. 1545-1547.

47. - Applications continues commutant avec les éléments d'un groupe de Lie, ibid. pp. 1784-1786.

48. - L'homologie d'un espace fibré dont la fibre est connexe, J. Math. Pures Appl. vol. 29 (1950) pp. 169-213.

49. - - Sur l'homologie des groupes de Lie, des espaces homogènes et des espaces fibrés principaux, Colloque de Topologie Algébrique, Bruxelles, 1950, Liège et Paris, 1951, pp. 101-115.

50. A. Lichnerowicz, Variêtés pseudo-kähleriennes à courbure de Ricci non nulle; application aux domaines bornés de $C^{n}, \mathrm{C}$. R. Acad. Sci. Paris vol. 235 (1952) pp. 12-14.

51. - - Sur les espaces homogènes kähleriens, ibid. vol. 237 (1953) pp. 695-697.

52. — Espaces homogènes kähleriens, Colloque de Géométrie différentielle, Strasbourg, 1953, Publ. du C.N.R.S. Paris, 1953, pp. 171-184.

53. - Un théorème sur les espaces homogènes complexes, Archiv der Mathematik vol. 5 (1954) pp. 207-215.

54. A. Malcev, On a class of homogeneous spaces, Izvestiya Akademii Nauk SSSR Ser. Math. vol. 13 (1949) pp. 9-32, translated in Amer. Math. Soc. Translation no. $42,1951$.

55. Y. Matsushima, On the discrete subgroups and homogeneous spaces of nilpotent Lie groups, Nagoya Math. Jour. vol. 2 (1951) pp. 95-110.

56. C. E. Miller, The topology of rotation groups, Ann. of Math. vol. 57 (1953) pp. 95-110.

57. G. D. Mostow, Factor spaces of solvable groups, Ann. of Math. vol. 60 (1954) pp. 1-27.

58. - - On covariant fiberings of Klein spaces, Amer. J. Math. vol. 77 (1955),

59. - Decomposition theorems for semi-simple Lie groups, Memoirs of the American Mathematical Society, no. 14, 1955.

60. K. Nomizu, On the cohomology of compact homogeneous spaces of nilpotent Lie groups, Ann. of Math. vol. 59 (1954) pp. 531-538.

61. J. Nordon, Les éléments d'homologie des quadriques et des hyperquadriques, Bull. Soc. Math. France vol. 74 (1946) pp. 11-129.

62. L. S. Pontrjagin, Homologies in compact Lie groups, Math. Sbornik (N.S.) vol. 6 (1939) pp. 389-422.

63. - Ueber die topologische Struktur der Lie'schen Gruppen, Comment Math. Helv. vol. 13 (1940-1941) pp. 227-238.

64. - Characteristic cycles on differentiable manifolds, Mat. Sbornik (N.S.) vol. 21 (1947) pp. 233-284. 
65. I. Z. Rosenknop, Homology groups of homogeneous spaces, C. R. Acad. Sci. URSS (N.S.) vol. 85 (1952) pp. 1219-1221.

66. H. Samelson, Beiträge zur Topologie der Gruppen-Mannigfaltigkeiten, Ann. of Math. vol. 42 (1941) pp. 1091-1137. $2-37$.

67. — , Topology of Lie groups, Bull. Amer. Math. Soc. vol. 58 (1952) pp.

68. - - A class of complex analytic manifolds, Portugaliae Math. vol. 12 (1953) pp. 129-132.

69. I. Satake, On a theorem of E. Cartan, J. Math. Soc. Japan vol. 2 (1951) pp. 284-305.

70. J-P. Serre, Groupes d'homotopie et classes de groupes abéliens, Ann. of Math. vol. 58 (1953) pp. 258-294.

71. - Quelques calculs de groupes d'homotopie, C. R. Acad. Sci. Paris vol. 236 (1953) pp. 2475-2477.

72. G. C. Shephard, On finite groups generated by reflections, Enseignement Mathematique, to appear. 14.

73. N. Steenrod, The topology of fibre bundles, Princeton Mathematical Series, no.

74. E. Stiefel, Ueber eine Beziehung zwischen geschlossenen Lie'schen Gruppen und diskontinuerlichen Bewegungsgruppen Euklidischer Räume und ihre Anwendung auf die Aufzählung einfacher Lie'scher Gruppen, Comment Math. Helv. vol. 14 (1941-1942) pp. $350-380$.

75. M. Sugawara, On the homotopy groups of rotation groups, Math. J. Okayama Univ. vol. 3 (1953-1954) pp. 11-21.

76. - Some remarks on homotopy groups of rotation groups, ibid. pp. 129-133.

77. J. Tits, Etude géométrique d'une classe d'espaces homogènes, C. R. Acad. Sci. Paris vol. 239 (1954) pp. 466-468.

78. - Sur les R-espaces, ibid. pp. 850-852.

79. H. C. Wang, Closed manifolds with homogeneous complex structure, Amer. J. Math. vol. 76 (1954) pp. 1-32.

80. - Complex parallisable manifolds, Proc. Amer. Math. Soc. vol. 5 (1954) pp. 771-776.

81. H. Weyl, Theorie der Darstellung kontinuerlicher halb-einfacher Gruppen durch lineare Transformationen, I, II, III, Math. Zeit. vol. 23 (1925) pp. 271-309, vol. 24 (1926) pp. 328-395.

82. Chih-Tah Yen, Sur les polynomes de Poincaré des groupes de Lie exceptionnels, C. R. Acad. Sci. Paris vol. 228 (1949) pp. 628-630.

InstituTE FOR AdVANCEd STUdy 